\title{
Soft X-ray properties of a spectroscopically selected sample of interacting and isolated Seyfert galaxies ${ }^{\star}$
}

\author{
F. Pfefferkorn ${ }^{1}$, Th. Boller ${ }^{1}$, and P. Rafanelli ${ }^{2}$ \\ 1 Max-Planck-Institut für extraterrestrische Physik, Postfach 1312, 85741 Garching, Germany \\ 2 Department of Astronomy, University of Padova, Vicolo Osservatorio 5, 35122 Padova, Italy
}

Received 12 October 2000 / Accepted 4 January 2001

\begin{abstract}
We present a catalogue of ROSAT detected sources in the sample of spectroscopically selected Seyfert 1 and Seyfert 2 galaxies of Rafanelli et al. (1995). The catalogue contains 102 Seyfert 1 and 36 Seyfert 2 galaxies. The identification is based on X-ray contour maps overlaid on optical images taken from the Digitized Sky Survey. We have derived the basic spectral and timing properties of the X-ray detected Seyfert galaxies. For Seyfert 1 galaxies a strong correlation between photon index and X-ray luminosity is detected. We confirm the presence of generally steeper X-ray continua in narrow-line Seyfert 1 galaxies (NLS1s) compared to broad-line Seyfert 1 galaxies. Seyfert 2 galaxies show photon indices similar to those of NLS1s. Whereas a tendency for an increasing X-ray luminosity with increasing interaction strength is found for Seyfert 1 galaxies, such a correlation is not found for Seyfert 2 galaxies. For Seyfert 1 galaxies we found also a strong correlation for increasing far-infrared luminosity with increasing interaction strength. Both NLS1s and Seyfert 2 galaxies show the highest values of far-infrared luminosity compared to Seyfert 1 galaxies, suggesting that NLS1s and Seyfert 2 galaxies host strong (circumnuclear) star formation. For variable Seyfert galaxies we present the X-ray light curves obtained from the ROSAT All-Sky Survey and from ROSAT PSPC and HRI pointed observations. Besides the expected strong shortand long-term X-ray variability in Seyfert 1 galaxies, we find indications for X-ray flux variations in Seyfert 2 galaxies.
\end{abstract}

Key words. catalogs - galaxies: active - galaxies: interactions - galaxies: Seyfert - X-rays: galaxies

\section{Introduction}

Interaction between galaxies is considered to have a wide importance in triggering starburst and AGN activity, which is thought to produce a rise in the luminosity.

Several studies of physical mechanisms which could trigger a burst of strong star formation have been investigated by Barnes \& Hernquist (1991, 1996), Jog \& Das (1992), Jog \& Solomon (1992) and Mihos \& Hernquist (1996).

Also many observations suggest that galactic encounters enhance star formation rates, as demonstrated by studies of the optical (e.g., Kennicutt et al. 1987; Bushouse 1987), infrared (e.g., Bernlöhr 1993; Telesco et al. 1993) and radio (e.g., Hummel 1981; Smith \& Kassim 1993) emission from interacting systems. In the X-ray band, an enhanced starburst activity will result in an increase in

Send offprint requests to: F. Pfefferkorn,

e-mail: pfefferk@mpe.mpg.de

* All overlays can be retrieved via CDS anonymous ftp to cdsarc.u-strasbg.fr (130.79.128.5) or via

http://cdsweb.u-strasbg.fr/cgi-bin/qcat?J/A+A/368/797 the X-ray luminosity due to the enhanced supernova rate. In addition, galaxy interaction might cause an increase in the accretion rate onto the black hole, resulting in an additional increase in the X-ray luminosity.

We have investigated the X-ray properties of a spectroscopically selected sample of Seyfert 1, NLS1 and Seyfert 2 galaxies with $z<0.11, m_{\mathrm{v}} \leq 15.5$ and $\delta \geq$ $-23^{\circ}$ (Rafanelli et al. 1995). The physical pairs have been selected on POSS plates using the following criteria: separation between components $S<3 D_{\mathrm{p}}$, where $D_{\mathrm{p}}$ is the apparent major axis of the Seyfert galaxy, and magnitude-difference between Seyfert galaxy and companion $\Delta m_{\mathrm{v}}=m_{\mathrm{v}, \mathrm{comp}}-m_{\mathrm{v}, \text { Seyfert }}<3$ (Rafanelli et al. 1995). Approximately $30 \%$ of the ROSATdetected sources within the Rafanelli sample are Seyfert galaxies with separated physical companions, 32 Seyfert 1 and 11 Seyfert 2 galaxies match the criteria mentioned above. Some of the isolated sources with a disturbed morphology are probably merging galaxies or very close pairs, for which no separation $S$ is given by Rafanelli et al. (1995). 
The aim of this paper is to present the timing and spectral X-ray properties of Seyfert 1, NLS1 and Seyfert 2 galaxies. In Sect. 2 we describe the X-ray data analysis as well as the identification process. The spectral features and timing results, including the relation between the interaction strength $Q$ and the X-ray luminosity $L_{\mathrm{X}}$, are discussed in Sect. 3. The summary is given in Sect. 4. The database of X-ray detected Seyfert galaxies is quoted in the Appendix C. The X-ray light curves of variable Seyfert 1 galaxies are presented in Figs. C. 1 to C.5 of the Appendix C.

\section{ROSAT detected interacting and isolated Seyfert $1 \& 2$ galaxies}

\subsection{Identification procedure}

For each source of the Rafanelli sample we have searched for X-ray detections within the ROSATAll-Sky Survey (RASS II catalogue; see Voges et al. 1999 for references) and public PSPC ${ }^{1}$ and $\mathrm{HRI}^{2}$ pointed observations. The sources have been identified on X-ray contour maps overlaid to optical images taken from the Palomar Digitized Sky Survey (DSS). We have generated background subtracted contour maps for each selected ROSATobservation available for inspection as postscript files at http://wave .xray .mpe.mpg.de/publications/papers/2001. These files can also be retrieved via anonymous ftp from the address ftp.xray.mpe.mpg.de ${ }^{3}$. Overlays with a dark background indicate pointed observations (HRI for HRI-data, without label for PSPC-data), whereas the grey background indicate ROSAT All-Sky survey data (PSPC). The quality of the identification of the X-ray source with the Seyfert galaxy is given in Tables C.1 and C.2, 1 and 2 indicate high and low reliability of the identification, respectively.

\subsection{Data reduction of survey and pointed observations}

During the ROSATAll-Sky Survey the sources took from $\sim 5 \mathrm{~s}$ to $\sim 30 \mathrm{~s}$ to pass across the field of view of the PSPC detector on each orbit ( $\sim 96 \mathrm{~min})$. The difference in crossing time depends on the scan range over the field of view, which covers a circular area with a 57 ' 3 radius. We have only counted crossing times of individual sources through the PSPC detector, when the centroid source position was at least $5^{\prime}$ inside the detector. This prevents an underestimate of the source count rate due to the PSF. The resulting total exposure times, obtained taking the sum of crossing times of a source, are in the range of $\sim 200 \mathrm{~s}$ up to $\sim 2000 \mathrm{~s}$ spread over several days. For the ROSATAll-Sky Survey data reduction we have developed our own software modules, to take into account the requirements for data reduction of the survey scan mode.

\footnotetext{
${ }^{1}$ Position Sensitive Proportional Counter.

${ }^{2}$ High Resolution Imager.

3 Subdirectory: publications/papers/2001/interacting-xray-seyferts
}

The data analysis of pointed ROSATPSPC and HRI observations was performed with the standard MIDAS/EXSAS software (Zimmermann et al. 1994). For this type of observation, the source is stationary centered on the detector. The exposure times are in general significantly higher with respect to the survey observations (see Tables C.3 and C.4 in Appendix C).

\subsection{Spectral analysis}

For each source with more than 60 photons detected in the All-Sky Survey and for all sources within PSPC-pointed observations we have performed a spectral analysis. A power-law model

$f_{\mathrm{Ed}} E \propto E^{\Gamma+1} \mathrm{~d} E$

fitted to the spectral data yields the power-law parameters; neutral absorbing hydrogen column density $N_{\mathrm{H}_{\text {fit }}}$, photon index $\Gamma$ and the monochromatic flux at $1 \mathrm{keV}$. The term $f_{\mathrm{E}} \mathrm{d} E$ is the galaxy's energy flux between the photon energies $E$ and $E+\mathrm{d} E$. The soft X-ray flux in the energy range $0.1-2.4 \mathrm{keV}$, corrected for absorption by neutral hydrogen, was calculated using the spectral fit parameters. In the case of $N_{\mathrm{H}_{\mathrm{fit}}}<N_{\mathrm{H}_{\mathrm{gal}}}$, we have used the galactic absorption column density $N_{\mathrm{H}_{\text {gal }}}$ (Dickey \& Lockman 1990).

For sources with $\leq 60$ counts in the survey observations and for HRI observations we have converted the mean count rates to the flux using a power-law model with photon index fixed to $\Gamma=-2.3$, which is the typical value found for extragalactic objects with ROSAT, and the galactic hydrogen column density $N_{\mathrm{H}_{\text {gal }}}$.

The soft X-ray flux was converted to luminosity using Eq. (7) of Schmidt \& Green (1986):

$L\left(E_{1}, E_{2}\right)=4 \pi\left(\frac{c}{H_{0}}\right)^{2} C(z, \Gamma) A(z)^{2} f\left(E_{1}, E_{2}\right)$

where a power-law spectrum is assumed in the energy range $\left(E_{1}, E_{2}\right)$, so that the redshift-dependent functions $C(z)$ and $A(z)$ are given by:

$C(z, \Gamma)=(1+z)^{\Gamma-2}$

$A(z)=2[(1+z)-\sqrt{1+z}]$.

We adopted for the cosmological deceleration parameter $q_{0}=\frac{1}{2}$ and for the Hubble constant $H_{\mathrm{o}}=75 \mathrm{~km} \mathrm{~s}^{-1} \mathrm{Mpc}^{-1}$. The assumed photon index $\Gamma$ is given in Tables C. 3 and C.4 and the redshift in Tables C.1 and C.2.

\subsection{Timing analysis}

We have compared the survey and pointed count rates of individual sources in order to study the long-term variability ( 0.5 up to 8.0 years). For all pointed observations 
and for the All-Sky Survey data with more than 60 source counts we have produced the corresponding X-ray light curves (timescales up to a few days).

To produce the light curve of a survey source ( $\geq 60$ counts), we have defined a source cell with radius of $5^{\prime}$. The background was determined from a source-free cell with a radius of $5^{\prime}$ located in the scan direction through the centroid position of the source. This was necessary, as the effective exposure time depends on the position of the scan direction and the background level may change for each time the path of source crosses the detector.

To take the ROSATwobble for pointed observations into account we have used a minimum binsize of $400 \mathrm{~s}$. From the light curve we have computed the mean count rate of the Seyfert 1 and Seyfert 2 galaxies, which are given in Tables C.3 and C.4, respectively.

The light curves of variable Seyfert 1 galaxies are given in Appendix C.

\section{Results}

In this section we present the X-ray properties of interacting and isolated Seyfert 1 \& 2 galaxies. A description of the database is given in Sect. 3.1. The spectral properties are listed in Tables C.3 and C.4, respectively. The light curves for variable Seyfert 1 galaxies are shown in Figs. C.1 to C.5. The X-ray light curves of Seyfert 2 galaxies are given in Sect. 3.3.2. Relations between $\Gamma, L_{\mathrm{X}},\left(L_{\mathrm{fir}}\right)$, $Q$ and the Seyfert type are presented in Sect. 3.2. The correlation of the interaction strength $Q$ and the X-ray luminosity $L_{\mathrm{X}}$ is described in Sect. 3.2.2.

\subsection{The catalogue}

We have detected 91 out of 99 Seyfert 1 and 47 out of 98 Seyfert 2 galaxies of the Rafanelli sample in the ROSATX-ray band in pointed and/or All-Sky Survey observations. We have performed spectral analysis of the ROSATPSPC observations for sources with more than 60 counts $\mathrm{s}^{-1}$. The timing analysis has been performed for all sources. In this paper we only show light curves of sources with significant X-ray variability. Spectral information from the survey data could be obtained for 59 Seyfert 1 galaxies and only for one Seyfert 2 galaxy. The optical and X-ray properties of Seyfert $1 \& 2$ galaxies of this sample are listed in Tables C.1 \& C.2. The tables quote the name of the Seyfert galaxy (Col. 2), ROSAT name (Col. 3), redshift $z$ (Col. 4), diameter of the Seyfert galaxy $D_{\mathrm{p}}$ (Col. 5), diameter of the companion galaxy $D_{\text {c }}$ (Col. 6), separation between the components $S$ (Col. 7), dimensionless gravitational interaction strength $Q$ (Col. 8) (for description see Sect. 3.2.2) and the apparent visual magnitude $V$ (Col. 9). The values of $z$ and $V$ are taken from Veron-Cetty \& Veron catalogue (1991) and the units of $D_{\mathrm{p}}, D_{\mathrm{c}}$ and $S$ are mm (POSS plates) with a scale $\sim 13.4^{\prime \prime} / \mathrm{mm}$ (Rafanelli et al. 1995). Columns 10 and 11 show the quality of the X-ray identifications both in the pointing and the survey observations, the identi- fications labeled either with 1 or 2, 1 and 2 indicating high and lower degree of reliability, respectively. In the last column we have listed the classification of the Seyfert type (Sy1.0, Sy1.2, Sy1.5, Sy1.8, Sy1.9, Sy2.0) taken from the Catalogue of Seyfert Galaxies (Lipovetski et al. 1987). We have modified the Rafanelli et al. conventions ( $\mathrm{S} 1=$ Sy1.0 + Sy1.2 + Sy1.5 and S2 $=$ Sy1.8 + Sy1.9 + Sy2.0) to $\mathrm{S} 1=\mathrm{Sy} 1.0+\mathrm{Sy} 1.2+\mathrm{Sy} 1.5+\mathrm{Sy} 1.8+\mathrm{Sy} 1.9$ and $\mathrm{S} 2=$ S2.0. The classifications marked by a hash (\#) indicate Narrow Line Seyfert 1 galaxies (NLS1) (e.g. Osterbrock et al. 1985; Boller et al. 1996; Grupe 1996).

In Appendix $\mathrm{C}$ the X-ray properties obtained from the timing and spectral analysis are listed in Tables C.3 and C.4. Columns 2 and 3 contain the ROSATposition. We mostly give the centroid source position from the pointed observation with the higher exposure times. The Cols. 4 and 5 list the count rates, Cols. 6 and 7 the corresponding exposure times, Cols. 8 and 9 the fluxes and Cols. 10 and 11 the luminosities of the sources detected in ROSATpointing and survey observations, respectively. The survey count rates were taken from the RASS II catalogue and the pointing count rates were computed from the light curves of the sources. The apices $p$ and $h$ in Col. 4 indicate that the source data are taken from a PSPC or HRI observation. In the Cols. 8 and 9 we apply the apices $f$ and $c$ to mark the data produced by spectral fit or by count rates. This specification applies also for Cols. 10 and 11. The Galactic column density is given in Col. 12 (Dickey \& Lockman 1990), while the column density obtained from the spectral fit is given in Col. 13. The other spectral fit parameters, namely the monochromatic flux at $1 \mathrm{keV}$ and the photon index are also given in Cols. 14 and 15 , respectively. The value $\Gamma=-2.3$ was used, if no reliable spectral fit could be obtained. When spectral information was available from the survey as well as from the pointed data, we quote the results from the pointed observations.

In most cases for optically separated close pairs, we detected in the X-ray band an unresolved single source (see overlays ${ }^{4}$ ). The results of these spectral fits are listed in Tables C.3 and C.4. When we detected two separate X-ray components, we created two spectra and we show the sum of the count rates, fluxes and luminosities and the single fit parameters $\left(N_{\mathrm{H}}, f_{1 \mathrm{keV}}, \Gamma\right)$ of the Seyfert galaxy in the tables.

\subsection{Relations between $\Gamma, L_{\mathrm{X}}, L_{\mathrm{fir}}, Q$ and Seyfert type}

In this section we present the spectral properties of Seyfert 1 and Seyfert 2 galaxies in terms of the relations between the photon index $\Gamma$, the interaction strength $Q$, the X-ray luminosity $L_{\mathrm{X}}$, the far-infrared luminosity $L_{\mathrm{fir}}$ and the Seyfert type.

\footnotetext{
${ }^{4}$ Overlays are available at: http://wave.xray.mpe.mpg.de/publications/papers/2001
} 


\subsubsection{Relations with $\Gamma$}

In Fig. 1 we have correlated the photon index obtained from the power-law fit with the X-ray luminosity. Different subtypes of Seyfert 1 galaxies are marked with different labels. For low-luminosity Seyfert 1's, below about $10^{42} \mathrm{erg} \mathrm{s}^{-1}$, where a significant contribution from the starburst is expected to contribute to the total luminosity, there is no clear trend between $\Gamma_{\text {fit }}$ and $L_{\mathrm{X}}$. However, for "normal" Seyfert 1 type galaxies, a clear trend of a steepening of the X-ray spectrum with increasing X-ray luminosity is detected. A possible explanation for this effect might be a shifted and strengthened accretion disk spectrum in high-luminosity Seyferts. This is expected, as the high X-ray luminosity is most probably related to the accretion rate and/or the black hole mass (Frank et al. 1985). When fitting a simple-power law to the spectral data in the ROSAT energy band, steeper values for the photon index are expected to arise in the high luminosity Seyfert 1 galaxies. Another well-known effect is also present in Fig. 1, i.e. the steeper X-ray continua of NLS1s compared to broad-line Seyfert 1 galaxies (Boller et al. 1996).

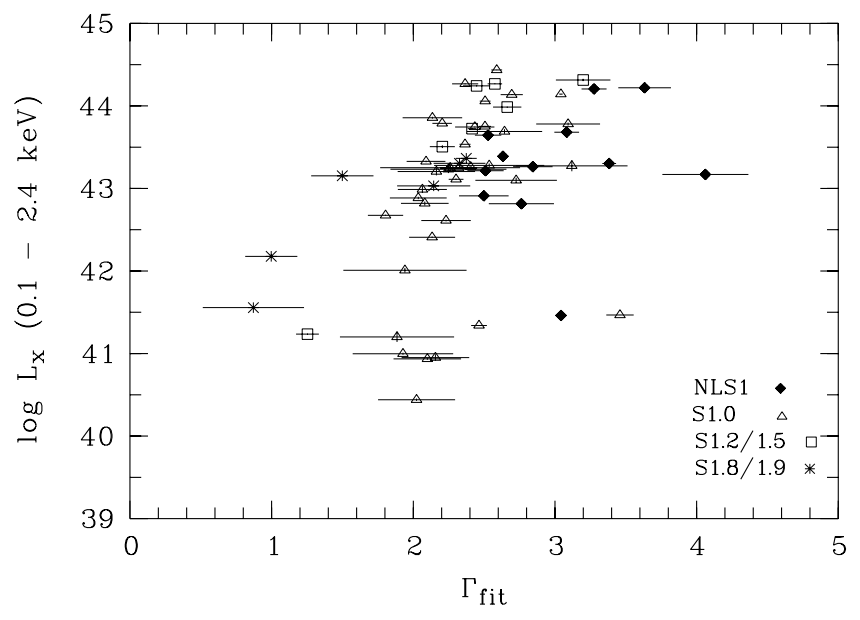

Fig. 1. Relation between the X-ray luminosity $L_{\mathrm{X}}\left[\mathrm{erg} \mathrm{s}^{-1}\right]$ and the photon index for Seyfert 1 galaxies. The different subtypes of Seyfert 1 galaxies (NLS1s, Seyfert 1.x) are plotted with different symbols. For Seyfert 1 galaxies above a X-ray luminosity of about $10^{42} \mathrm{erg} \mathrm{s}^{-1}$ we find a strong trend of an increasing photon index with increasing X-ray luminosity.

For Seyfert 2 galaxies, we found no significant trend for an increasing photon index with increasing X-ray luminosity. Higher sensitivity measurements, e.g. with XMMNewton, are necessary to search for a correlation between the photon index and the X-ray luminosity for Seyfert 2 galaxies.

In Fig. 2 we show the distribution of photon indices, obtained from the spectral fits, versus the Seyfert type (we have plotted NLS1s at an $x$-axis value of 0.9 ). Only Seyfert galaxies with errors in the photon index smaller than 0.5 have been included. As expected, NLS1s show the largest values of the photon index, compared to Seyfert 1 galaxies. Seyfert 2 galaxies show similar steep X-ray continua compared to NLS1s. The Seyfert 1 galaxies show similar values of the photon indices as given by Walter \& Fink (1993).

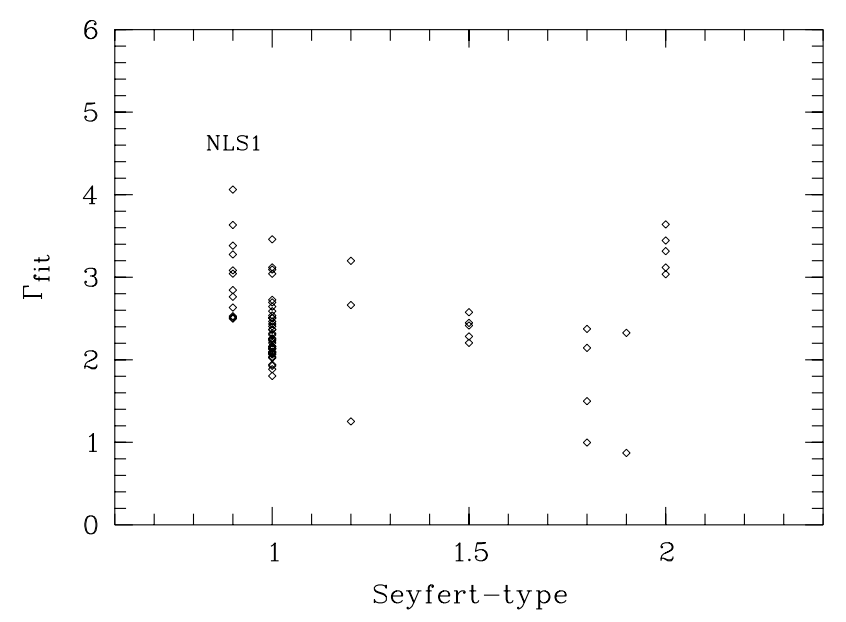

Fig. 2. Distribution of photon indices, obtained from the spectral fits, versus the Seyfert type (we have plotted NLS1s at an $x$-axis value of 0.9 ). The highest values in the photon indices are found for NLS1s and for Seyfert 2 galaxies

\subsubsection{Interaction strength $Q$ - luminosity $L_{\mathrm{X}}, L_{\mathrm{fir}}$ relations}

In order to derive the interaction strength $Q$ we concentrate on the tidal force per unit mass produced by a companion on a primary galaxy, which is proportional to $M_{\mathrm{c}} \cdot R^{-3} \cdot M_{\mathrm{c}}$ is the mass of the companion and $R$ is its distance from the center of the primary galaxy. In most cases, $M_{\mathrm{c}}$ and the absolute value of $R$ are unknown. Instead, these parameters are related to the dimensions of the pair. Rubin et al. (1982) describe the dependence of the mass $M$ of a galaxy on the size of its major axis $d$ as $M \propto d^{\gamma}$ and we use $\gamma=1.5$ (Dahari 1984). If we use the apparent diameter of the primary galaxy $D_{\mathrm{p}}$ as a scaling factor, we obtain:

$M_{\mathrm{c}} \propto\left(D_{\mathrm{c}} / D_{\mathrm{p}}\right)^{1.5} \quad$ and $\quad R \propto S / D_{\mathrm{p}}$

Using these relations we get as dimensionless gravitational interaction strength $Q$ :

$\frac{M_{\mathrm{c}}}{R^{3}} \propto \frac{\left(D_{\mathrm{c}} \cdot D_{\mathrm{p}}\right)^{1.5}}{S^{3}} \equiv Q$.

This parameter is obviously large for close and relatively large companions.

Figure 3 shows the interaction strength $Q$ vs. the farinfrared luminosity $L_{\text {fir }}$ for Seyfert 1 galaxies. For Seyfert 1 galaxies, the far-infrared luminosity increases with interaction strength. The low-luminosity Seyfert 1 galaxies NGC 5273, NGC 4278, NGC 3227, (NGC 4258 is 
not detected in the IRAS Faint Source Catalogue) also show the trend of increasing X-ray and far-infrared luminosity with interaction strength $\mathrm{Q}$. We speculate a large interaction strength of $Q=15.1 \pm 4.4$ for the galaxy Mkn 1040 causes the relatively high X-ray (see Fig. 4) and far-infrared luminosity and that the galaxy belongs to the low-luminosity population discussed above. To test the correlation we have calculated the linearcorrelation coefficient $r$ and the probability $P(r, N)$ for a linear correlation. For the high-luminosity objects we obtain $r=0.3271$ and $P(r, 22) \approx 85 \%(\sim 1.5 \sigma)$, whereas we got for the low-luminosity Seyferts $r=0.8478$ and $P(r, 4) \approx 85 \%(\sim 1.5 \sigma)$.

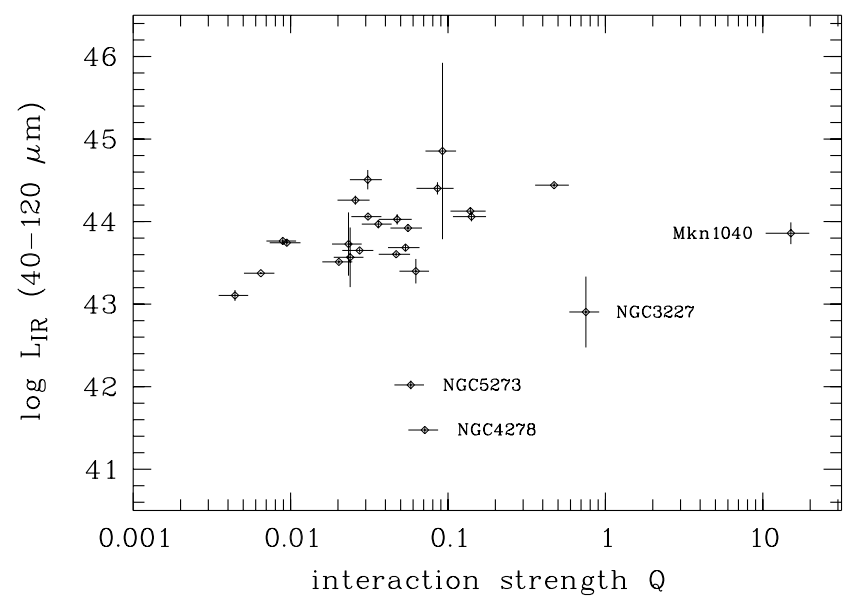

Fig. 3. Dimensionless gravitational interaction strength $Q$ vs. far-infrared luminosity $L_{\text {fir }}\left[\mathrm{erg} \mathrm{s}^{-1}\right]$ for Seyfert 1.x galaxies. The plot suggests an increase in luminosity for increasing values of the interaction strength

Figure 4 shows the interaction strength $Q$ vs. the soft $\mathrm{X}$-ray luminosity $L_{\mathrm{X}}$ for Seyfert 1 galaxies. For Seyfert 1 galaxies with $L_{\mathrm{X}}>10^{42}$ there is a tendency for a luminosity increase with increasing interaction strength. The labelled sources refer to the low-luminosity Seyfert 1 galaxies in our sample. As discussed for the relation between the far-infrared luminosity and the interaction strength $Q$, both the low-luminosity Seyfert 1's and the highluminosity Seyfert 1's increase in X-ray luminosity when the interaction strength $Q$ is increased. The high spread of the distribution is quite likely produced by an overlap of effects from starburst and AGN. In the case of infrared luminosity (Fig. 3) only the starburst play a role. The linear-correlation test resulted in $r=0.2880$ and $P(r, 27) \approx 85 \%(\sim 1.5 \sigma)$ for the high-luminosity Seyferts and in $r=0.8233$ and $P(r, 5) \approx 92 \%(\sim 1.7 \sigma)$ for the low-luminosity objects.

The data points in Fig. 4 also include ROSAT pointed observations and Seyfert 1.8 and 1.9 galaxies, which complete the relation between $L_{\mathrm{X}}$ and $Q$ first discussed by Rafanelli et al. (1997). For Seyfert 2 galaxies we found no correlation between the soft X-ray luminosity and the interaction strength. In Sect. 4 we discuss the problems

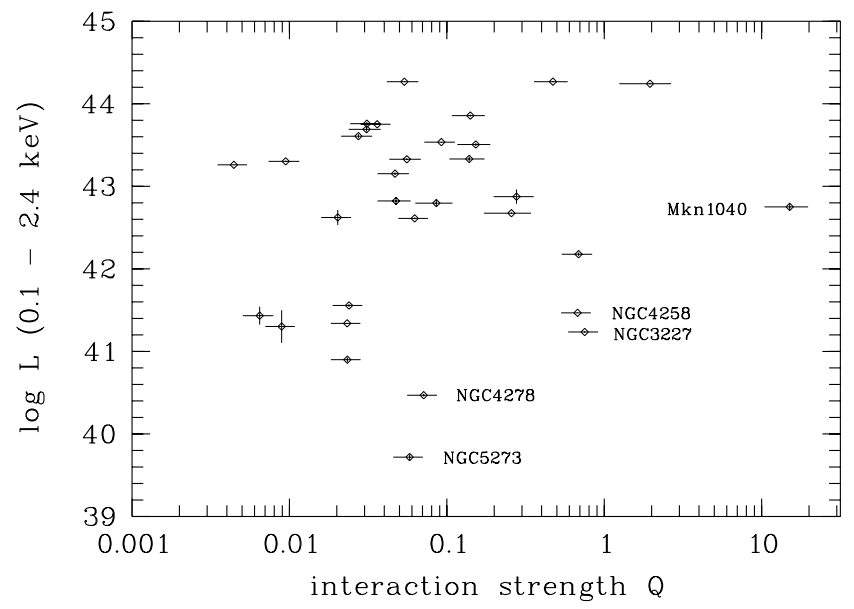

Fig. 4. Dimensionless gravitational interaction strength $Q$ vs. soft X-ray luminosity $L_{\mathrm{X}}\left[\mathrm{erg} \mathrm{s}^{-1}\right]$ for Seyfert $1 . x$ galaxies. The plot suggests an increase in luminosity for increasing values of the interaction strength. The marked objects with low luminosities are probably absorbed sources with emission from the circumnuclear starbursts and scattered radiation from the nuclei

in determining precisely the X-ray properties of obscured Seyfert 2 galaxies.

\subsubsection{Far-infrared relations}

In order to estimate the starburst activity we have investigated the far-infrared luminosity $L_{\text {fir }}$ using the farinfrared fluxes $f_{\text {fir }}$ at 60 and $100 \mu \mathrm{m}$ from the IRAS Faint Source Catalogue. The total far-infrared fluxes $f_{\text {fir }}(40-$ $120 \mu \mathrm{m})$ were computed following Helou (1985) from the IRAS $60 \mu \mathrm{m}$ and $100 \mu \mathrm{m}$ band fluxes:

$f_{\text {fir }}=1.2610^{-11}\left(2.58 f_{60}+f_{100}\right) \mathrm{erg} \mathrm{cm}^{-2} \mathrm{~s}^{-1}$

where $f_{60}$ and $f_{100}$ are given in Jansky. The far-infrared fluxes were converted to luminosities using Eq. (2) in Sect. 2.3. For the photon index we assumed $\Gamma=1.5$.

The ratio between far-infrared and soft X-ray luminosity $L_{\mathrm{X}} / L_{\text {fir }}$ and its dependence on the far-infrared luminosity $L_{\text {fir }}$ is shown in Fig. 5 for all Seyfert types. Interestingly, NLS1 galaxies show a similar distribution of the far-infrared luminosity as the Seyfert 2 galaxies. The far-infrared luminosity distribution is significantly different for Seyfert 1 galaxies compared to NLS1 and Seyfert 2's.

In Fig. 6 we plot the ratio $L_{\mathrm{X}} / L_{\mathrm{fir}}$ vs. the interaction strength $Q$ for all Seyfert types. For Seyfert 1 galaxies no correlation between the luminosity ratio and the interaction strength is found. If we can interprete the far-infrared luminosity as mainly caused by starburst activity, and the X-ray luminosity as mainly caused by accretion processes, this indicates that starburst and AGN activity increase proportionally.

The interpretations of the results are discussed in Sect. 4. 


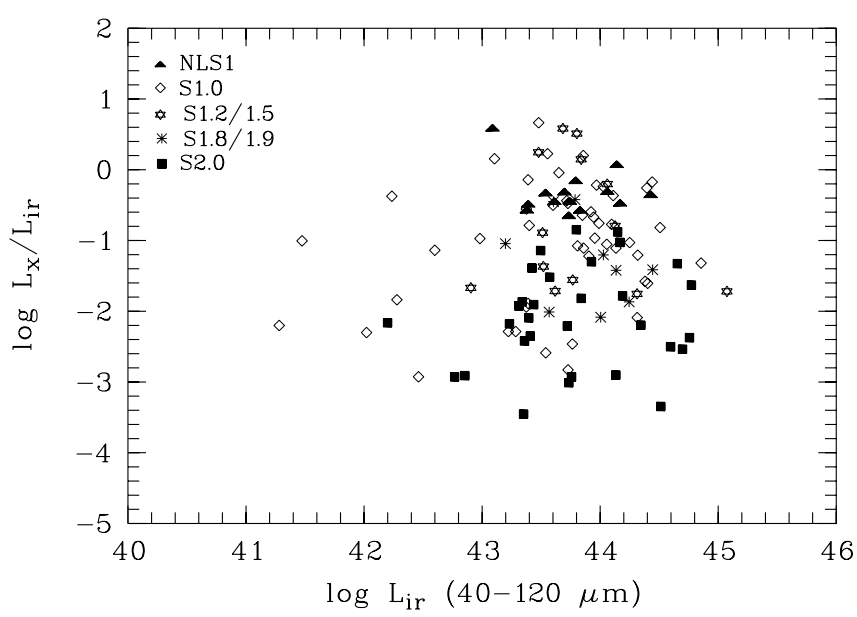

Fig. 5. Soft X-ray to far-infrared $(40-120 \mu \mathrm{m})$ luminosity ratio $L_{\mathrm{X}} / L_{\text {fir }}$ vs. far-infrared luminosity $L_{\text {fir }}\left[\mathrm{erg} \mathrm{s}^{-1}\right]$ for all types of Seyfert galaxies

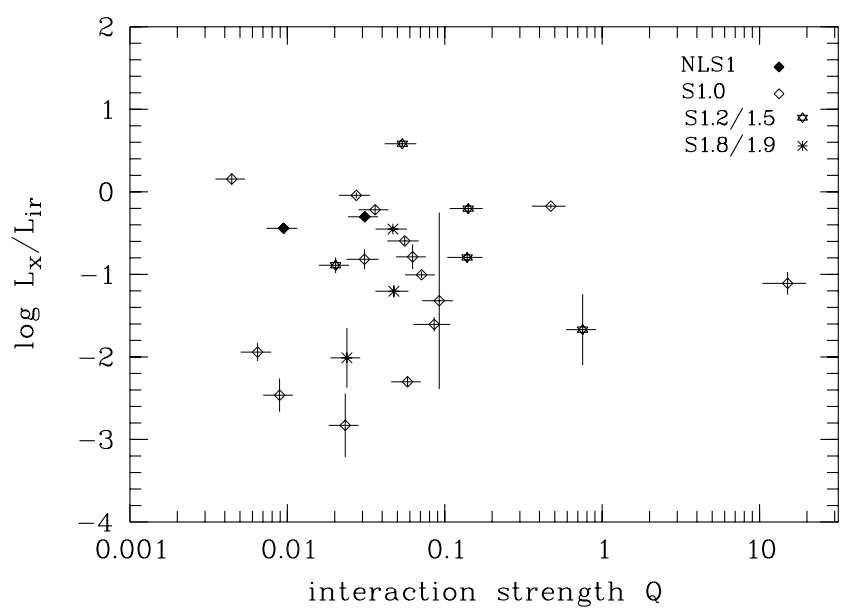

Fig. 6. Soft X-ray to far-infrared $(40-120 \mu \mathrm{m})$ luminosity ratio $L_{\mathrm{X}} / L_{\text {fir }}$ vs. dimensionless gravitational interaction strength $Q$ for all types of Seyfert 1.x galaxies. There is no correlation noticeable

\subsection{Timing properties}

Below we discuss the long-term (time scales between half a year up to 8 years) X-ray and short-term (time scales of order up to a few days) variability of interacting and isolated Seyfert 1 and 2 galaxies.

\subsubsection{X-ray variable Seyfert 1 galaxies}

In our sample $59 \%$ of the X-ray detected Seyfert 1 galaxies show significant X-ray variability during the ROSAT AllSky Survey and ROSAT pointed observations. The corresponding X-ray light curves are shown in Appendix C.

In Fig. 7 we compare the ROSAT All-Sky Survey count rate with the count rate measured in ROSAT PSPC pointed observations. The most extreme factor of vari-

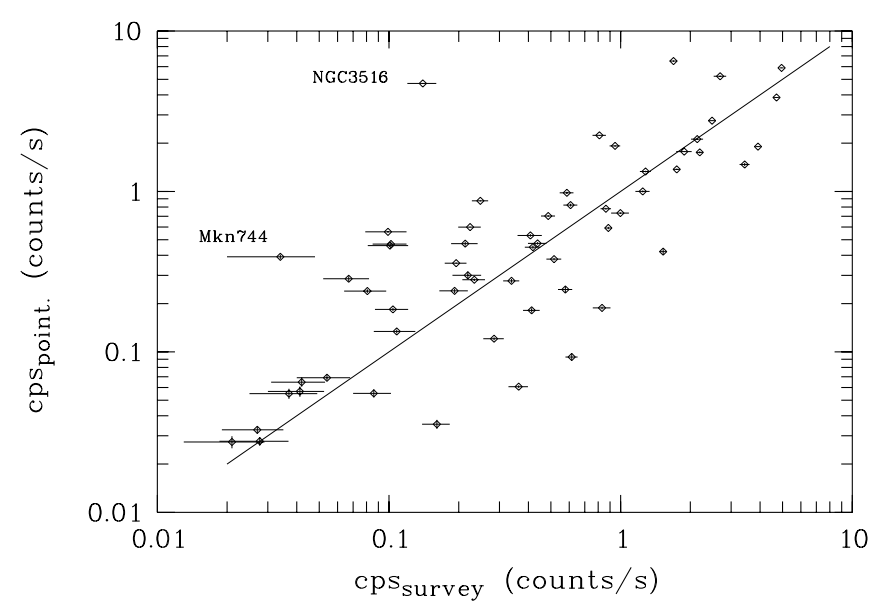

Fig. 7. Long-term variability of interacting and isolated Seyfert 1 galaxies. The most extreme factor of amplitude variability of about 33 is found for NGC 3516

ability is found for NGC 3516 (a factor of about 33 on a timescale of 718 days).

\subsubsection{The X-ray light curves for Seyfert 2 galaxies}

For interacting and isolated Seyfert 2 galaxies no indication for significant X-ray variability on timescales above 0.5 years is found by comparing the ROSAT All-Sky Survey and ROSAT PSPC pointed observations (Fig. 8). The galaxy NGC 5506 is classified by Lipovetski et al. (1987) as Seyfert type 2. This source exhibits the largest factor of variability of about 2.7 on a timescale of 375 days.

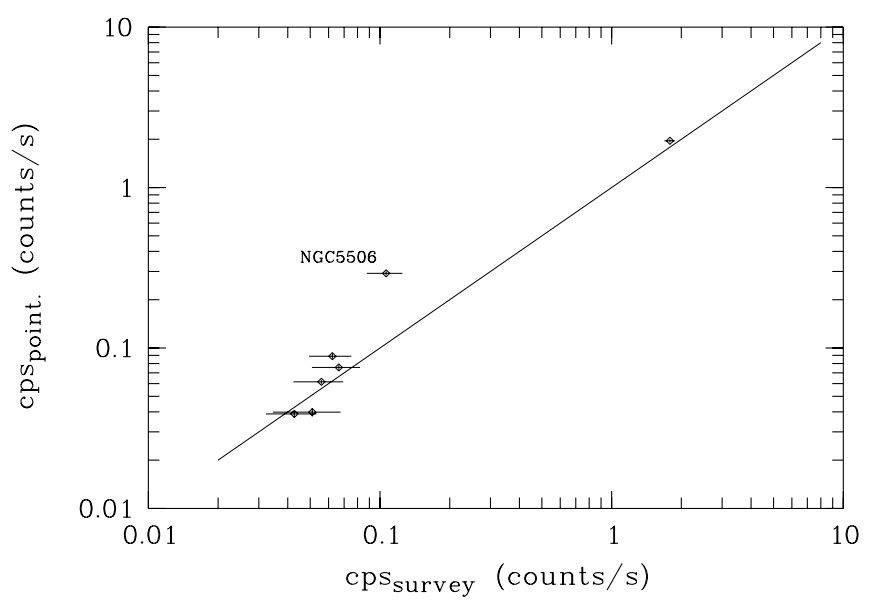

Fig. 8. The long-term variability of interacting and isolated Seyfert 2 galaxies

However, for three out of the 36 Seyfert 2 galaxies, NGC 1068, NGC 4388 and IRAS F01475-0740, indications for X-ray variability are found in ROSAT pointed observations. In Fig. 9 (top) the pointed observation light curve of the Seyfert 2 galaxy NGC 1068 is shown. An increase in count rate from 1.823 to 2.080 counts $\mathrm{s}^{-1}$, 

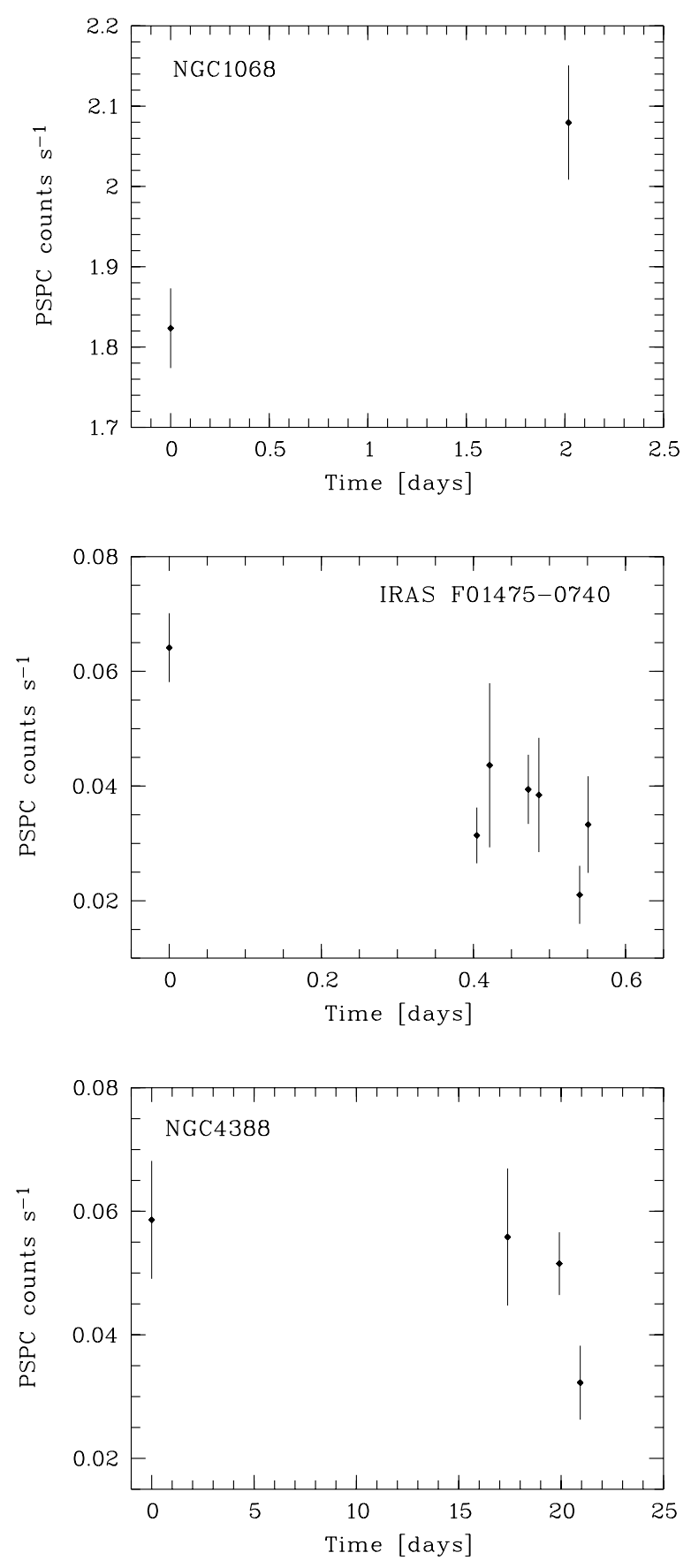

Fig. 9. PSPC pointed light curves of the Seyfert 2.0 galaxies NGC 1068 (top) with the probabilities of variability of

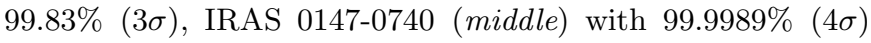
and NGC 4388 (bottom) with $97.896 \%(2 \sigma)$

corresponding to a factor of 1.14 or $\Delta \mathrm{cps}=0.256$, within 2 days is detected. A constant model fit using the $\chi^{2}$ test can be rejected with a probability of $99.83 \%$, corresponding to $3 \sigma$. Indications for X-ray variability in NGC 1068 are also found in other ROSATpointed observations (cf. Fig. B.1). The ROSAT PSPC light curve for the Seyfert 2 galaxy IRAS 01475-0748 is shown in Fig. 9 (middle). A

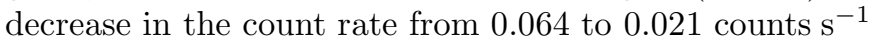

within 12.9 hours is detected. This variability corresponds to a factor of 3 and to a change in the count rate of $\Delta$ cps $=0.043$. A constant model fit gives a probability of $99.9989 \%(4 \sigma)$. In Fig. 9 (bottom) the X-ray light curve of NGC 4388 is shown. The count rate decreases from 0.0586 to 0.0322 counts $\mathrm{s}^{-1}$ corresponding to a factor of variability of about 1.8 and a change in the count rate of $\Delta \mathrm{cps}=0.026$ within 21 days. A constant model fit can be rejected with a probability of $97.896 \%$, corresponding to $2 \sigma$.

Recently, Georgantopoulos \& Papadakis (2000) found evidence for spectral (and timing) variability for four Seyfert 2 galaxies in RXTE observations.

\section{Discussion and summary}

We have detected $92 \%$ of interacting or isolated Seyfert 1 and $48 \%$ of Seyfert 2 galaxies in the optically selected sample of Rafanelli et al. (1995). The soft X-ray spectral and timing properties are presented in Tables C.3 and C.4 (note the different combination of Seyfert types; 102 S1.x and $36 \mathrm{~S} 2$ ).

For Seyfert 1 galaxies we have found a correlation between photon index $\Gamma$ and the soft X-ray luminosity $L_{\mathrm{X}}$. A clear trend of a steepening of the X-ray spectrum with increasing X-ray luminosity is detected. High $\mathrm{X}$-ray luminosity is most probably related to the accretion rate and/or the black hole mass. Therefore, a possible explanation for this effect might be a shifted and strengthened accretion disk spectrum in high-luminosity Seyferts. We confirm that NLS1s have steeper X-ray continua than broad-line Seyfert 1 galaxies. Seyfert 2 galaxies show similar steep X-ray continua compared to NLS1s. While the steep X-ray continua for NLS1s are expected to be related with high values for the Eddington luminosity in combination with small black hole masses, the steep $\mathrm{X}$-ray continua for Seyfert 2 galaxies are probably due to the dominant X-ray line emission from the circumnuclear starburst.

Our data result in an increasing far-infrared and increasing X-ray luminosity with increasing interaction strength for Seyfert 1 galaxies. Both strengthen the suggestion that galaxy interaction triggers an increased accretion rate and starburst rate. NLS1s and Seyfert 2 galaxies show the highest values of far-infrared luminosity compared to Seyfert 1 galaxies. This fact points to nuclear starburst activity taking place in NLS1 galaxies as suggested by Mathur (2000).

For Seyfert 2 galaxies, we found no significant correlations between the X-ray luminosity and photon index or interaction strength. The main reason is the lack of penetration through the high column densities in Seyfert 2 galaxies with soft X-rays. The ROSAT $N_{\mathrm{H}}$ values derived from the spectral fitting are therefore lower limits to the true absorbing columns in Seyfert 2 galaxies. This is supported by comparing our results with those from higher energy satellites. Bassani et al. (1999) 
investigated the hard X-ray spectra of a large sample of Seyfert 2 galaxies with Ginga, ASCA and BeppoSAX. The authors found a large population of strong-absorbed objects with column densities $N_{\mathrm{H}} \geq 10^{23} \mathrm{~cm}^{-2}$, including many Compton-thick candidates. The column densities obtained from the soft X-ray spectra show significantly lower values for many sources. The reason for this is the different origin of the hard and soft $\mathrm{X}$-ray radiation. The column densities are likely able to determine the outer regions of the molecular torus at soft X-rays, because the radiation penetrates less absorbing material. Only the hard $\mathrm{X}$-ray radiation above a few $\mathrm{keV}$ is able to pass interior regions of the torus and leads to higher column densities. Therefore, our results for Seyfert 2 galaxies are only a lower limit to the intrinsic column density. The fluxes and luminosities of Seyfert 2 galaxies given in Table C. 4 are considered as lower limits to the intrinsic values. Finally, the soft X-ray emission of Seyfert 2 galaxies is probably due to emission from the circumnuclear starburst together with radiation from the nuclei, scattered and reflected by the molecular torus. Moreover, we found no correlation between the far-infrared luminosity and interaction strength for the Seyfert 2 sample.

Higher sensitivity observations with XMM-Newton and Chandra are expected to confirm the short-time variability of the three Seyfert 2 galaxies.

We have investigated the variability of Seyfert 1 and 2 galaxies on short and long timescales and found indications for variability in three Seyfert 2.0 galaxies on short timescales (NGC 1068, IRAS 0147-0740, NGC 4388). A possible explanation for this variability might be the presence of boreholes in the absorbing molecular torus around the central black hole region. Significant X-ray variability during the ROSATpointed and survey observations were detected for 58 percent of the Seyfert 1 galaxies.

Acknowledgements. We thank Prof. Joachim Trümper for comments and A. Vogler for help in producing the images with overlayed X-ray contours. We are grateful to Dr. W. Voges for his help in the catalogue preparation. The ROSATproject is supported by the Bundesministerium für Bildung und Forschung (BMBF/DLR) and by the Max-Planck Society (MPG).

This paper can be retrieved via WWW from our pre-print server:

http://wave.xray.mpe.mpg.de/publications/papers/2001

\section{Appendix A: Notes on individual sources}

- NGC 2992: In the case of NGC 2992 and its companion we detect two spatially separated X-ray components. In Table C. 4 of Appendix $\mathrm{C}$ we give the sum of the count rates, fluxes and luminosities for this system. For the completion of the database we add here the individual values of the source and the companion. The spectrum of NGC 2992 shows a high absorption and a low photon index with an integrated flux of $2.81510^{-12} \mathrm{erg} \mathrm{cm}^{-2} \mathrm{~s}^{-1}$ corresponding to a luminosity of $2.61610^{41} \mathrm{erg} \mathrm{s}^{-1}$. The spectrum of the companion results in a flux of $1.06110^{-12} \mathrm{erg} \mathrm{cm}^{-2} \mathrm{~s}^{-1}$ corresponding to a luminosity of $9.98710^{40} \mathrm{erg} \mathrm{s}^{-1}$. The spectral fit parameters for the companion are $N_{\mathrm{H}_{\text {fit }}}=0.85410^{21} \mathrm{~cm}^{-2}$ and $\Gamma=-2.72$.

NGC 2992 is classified by Lipovetski et al. (1987) as a Seyfert 1.9 galaxy and we found a variability of this object over short and long timescales.

- NGC 5506: The soft X-ray spectrum of the Seyfert galaxy NGC 5506 is highly absorbed and no reliable spectral fit parameters can be obtained. Thus, we have used for $N_{\mathrm{H}}=1.010^{21} \mathrm{~cm}^{-2}$ as a lower limit in Table C.4.

- NGC 5953: This interacting system (Rafanelli et al. 1990) is detected in the survey and pointed observations. The X-ray emission of this system in the pointed observation is mainly caused by the Seyfert 2 galaxy NGC 5953. In the survey observation the emission is centered on the companion of NGC 5953 and no emission from the Seyfert 2 galaxy is detected. Due to the PSPC pointing accuracy we are not able to decide whether NGC 5953 is a transient source in the soft $\mathrm{X}$-ray band.

- Mkn 684: For Mkn 684 we assume the classification as a NLS1 galaxy by Osterbrock \& Pogge (1985) and Grupe (1996).

- NGC 7319, a member of the Stefan's Quintet group, is detected with the PSPC (pointed and survey) and HRI detector by ROSAT. The PSPC survey and pointed observations showed unresolved X-ray emission from the group and the intergalactic gas (Pietsch \& Trinchieri 1997). The HRI pointed observation as used to determine the flux of the Seyfert 2 galaxy.

- NGC 3031: For NGC 3031 (M 81) we used the distance of $3.63 \mathrm{Mpc}$ given in Freedman et al. (1994) to compute the luminosity of this object $(z<0)$.

\section{Appendix B: Light curves of NGC 1068}

In Fig. B.1 we give the remaining PSPC and HRI light curves of the Seyfert 2 galaxy NGC 1068.

\section{Appendix C: The Catalogue - data tables and light curves}

In this section we show the results of our studies of the Xray properties of the Seyfert 1 and 2 galaxies in Tables C.3 and C.4, while in Tables C.1 and C.2, general and optical properties are listed. The light curves of variable Seyfert 1 galaxies are also summarized in this Appendix in Figs. C.1 to C.5. To distinguish the survey from the pointing light curves we have labeled the survey curves. The pointed light curves are distinguished by the $y$-axis in PSPC or HRI based data. 

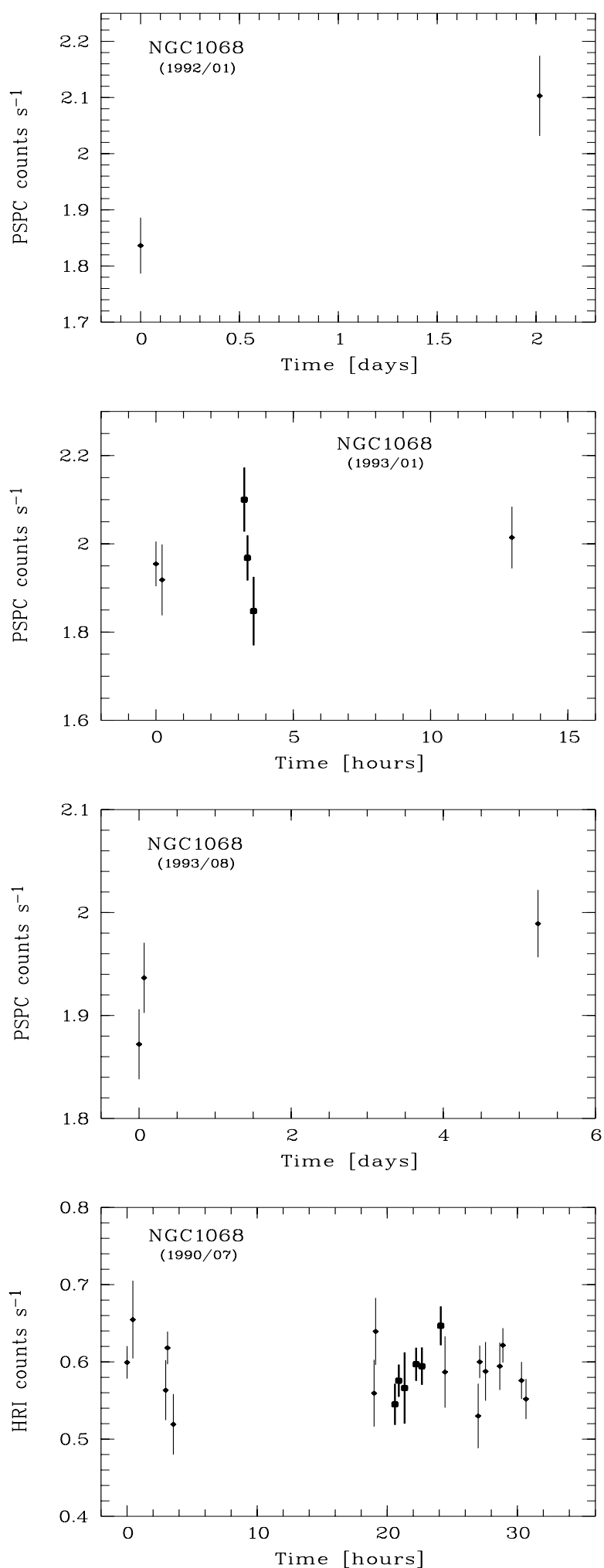

Fig. B.1. Pointing light curves of the Seyfert 2.0 galaxies NGC 1068 (from top to bottom): first panel: this PSPC light curve of NGC 1068 from 1992/01 was described in Sect. 3.3 .2 already. Second panel: The bold data points in the light curve of the PSPC observation from 1993/01 indicate a variability with a probability of $94.24 \%(\sim 2 \sigma)$. The count rate decrease from 2.10 to 1.85 counts s${ }^{-1}$ corresponds to a factor of 1.14 or $\Delta \mathrm{cps}=0.253$ within 1 hour. Third panel: the third PSPC pointing light curve of NGC 1068 shows a probability of variability of $95.47 \%(2 \sigma)$ with an increase of $\Delta \mathrm{cps}=0.117$ counts s$^{-1}$. Fourth panel: a HRI pointed light curve of this object indicates also a variability, with a probability of $88,82 \%$ for the bold data points. The count rate increase from 0.545 to 0.647 counts s$^{-1}$ within 3.5 hours correlates with a variability factor of 1.19 or $\Delta$ cps $=0.102$ 
Table C.1. Seyfert 1 galaxies: A part of the Rafanelli sample of interacting or isolated Seyfert 1 galaxies detected by ROSAT. The table contains optical and generical properties as well as some results from the investigations of the Rafanelli group (Rafanelli et al. 1995). Col. (2) - object name, Col. (3) - ROSAT name, Col. (4) - redshift, Cols. (5 and 6$)$ - diameter of Seyfert galaxy and companion, Col. (7) - distance between the components, Col. (8) - interaction strength, Col. (9) - apparent visual magnitude, Cols. (10 and 11) - quality of X-ray identification (1: high, 2: lower degree of reliability) and Col. (12) Seyfert type. The upper index \# in Col. (12) marks objects, which are classified as NLS1 galaxy (Osterbrock \& Pogge 1985; Boller et al. 1996; Grupe 1996)

\begin{tabular}{|c|c|c|c|c|c|c|c|c|c|c|c|}
\hline$\overline{\mathrm{Nr} .}$ & name & ROSAT name & $z$ & $\begin{array}{c}D_{\mathrm{p}} \\
\mathrm{mm}\end{array}$ & $\begin{array}{c}D_{\mathrm{c}} \\
\mathrm{mm}\end{array}$ & $\begin{array}{c}S \\
\mathrm{~mm}\end{array}$ & $\overline{\bar{Q}}$ & $\begin{array}{c}V \\
\mathrm{mag}\end{array}$ & point. & sur. & $\begin{array}{c}\text { Sy } \\
\text { type }\end{array}$ \\
\hline 1 & Mkn 334 & 1RXS J000308.6+215728 & 0.022 & 3.7 & - & - & - & 14.62 & - & 1 & 1.8 \\
\hline 2 & Mkn 335 & 1RXP J000619.2+201224 & 0.025 & 1.2 & - & - & - & 13.85 & 1 & 1 & 1.0 \\
\hline 3 & Mkn 1146 & 1RXS J004719.4+144215 & 0.039 & 2.6 & - & - & - & 15.28 & - & 1 & 1.0 \\
\hline 4 & Mkn 352 & 1RXP J005953.3+314947 & 0.015 & 1.4 & - & - & - & 14.66 & 1 & 1 & 1.0 \\
\hline 5 & Mkn 1152 & 1RXP J011350.1-145034 & 0.052 & 2.2 & 1.2 & 1.3 & $1.9524 \pm 0.7060$ & 15.00 & 1 & 1 & 1.5 \\
\hline 6 & Mkn 993 & 1RXS J012531.4+320800 & 0.017 & 7.5 & - & - & - & 13.96 & - & 1 & 1.9 \\
\hline 7 & Mkn 975 & 1RXS J011350.4+131534 & 0.050 & 2.0 & - & - & - & 14.50 & - & 1 & 1.5 \\
\hline 8 & Mkn 358 & 1RXS J012634.2+313659 & 0.042 & 2.8 & - & - & - & 15.23 & - & 1 & 1.0 \\
\hline 9 & Mkn 359 & 1RXP J012732.8+191051 & 0.017 & 2.6 & - & - & - & 14.21 & 1 & 1 & $1.5^{\#}$ \\
\hline 10 & Mkn 1018 & 1RXP J020616.0-001732 & 0.043 & 2.6 & - & - & - & 14.65 & 1 & 1 & 1.9 \\
\hline 11 & Mkn 590 & 1RXP J021433.7-004604 & 0.027 & 4.1 & 0.8 & 4.8 & $0.0537 \pm 0.0122$ & 13.55 & 1 & 1 & 1.5 \\
\hline 12 & Mkn 1040 & 1RXS J022814.6+311838 & 0.016 & 9.8 & 1.8 & 1.7 & $15.0800 \pm 4.6514$ & 14.74 & - & 1 & 1.0 \\
\hline 13 & Mkn 1044 & 1RXP J023005.8-085940 & 0.016 & 1.7 & - & - & - & 14.67 & 1 & 1 & $1.0^{\#}$ \\
\hline 14 & NGC 985 & 1RXP J023437.9-084709 & 0.043 & 3.2 & - & - & - & 14.95 & 1 & 1 & 1.0 \\
\hline 15 & Mkn 595 & 1RXS J024135.2+071117 & 0.028 & 1.7 & 1.0 & 2.0 & $0.2771 \pm 0.0790$ & 14.40 & - & 1 & 1.0 \\
\hline 16 & Mkn 372 & 1RXP J024920.6+191822 & 0.031 & 1.7 & - & - & - & 14.81 & 1 & 1 & 1.8 \\
\hline 17 & Mkn 609 & 1RXP J032525.5-060827 & 0.032 & 1.1 & - & - & - & 14.12 & 1 & 1 & 1.8 \\
\hline 18 & Mkn 618 & 1RXS J043622.3-102226 & 0.035 & 2.6 & 0.7 & 4.3 & $0.0309 \pm 0.0071$ & 14.65 & 1 & 1 & 1.0 \\
\hline 19 & Mkn 6 & 1RXS J065209.8+742537 & 0.019 & 1.8 & - & - & - & 14.02 & - & 1 & 1.5 \\
\hline 20 & Mkn 374 & 1RXS J065938.5+541136 & 0.044 & 1.6 & - & - & - & 14.38 & - & 2 & 1.2 \\
\hline 21 & Mkn 9 & 1RXS J073657.0+584610 & 0.039 & 1.7 & - & - & - & 14.68 & - & 1 & 1.0 \\
\hline 22 & Mkn 79 & 1RXP J074233.5+494838 & 0.022 & 3.3 & - & - & - & 14.78 & 1 & 1 & 1.2 \\
\hline 23 & Mkn 10 & 1RXP J074728.6+605552 & 0.030 & 5.2 & 2.1 & 10.0 & $0.0361 \pm 0.0078$ & 14.70 & 1 & 1 & 1.0 \\
\hline 24 & Mkn 382 & 1RXS J075526.1+391111 & 0.034 & 2.1 & - & - & - & 15.50 & 1 & 1 & 1.0 \\
\hline 25 & Mkn 1218 & 1RXP J083810.8+245336 & 0.028 & 2.6 & 1.3 & 5.1 & $0.0468 \pm 0.0105$ & 14.12 & 1 & 1 & 1.8 \\
\hline 26 & NGC 2639 & 1RXP J084338.0+501207 & 0.011 & 6.5 & - & - & - & 11.39 & 1 & - & 1.0 \\
\hline 27 & NGC 2782 & 1RXH J091405.1+400651 & 0.008 & 8.5 & 1.5 & 12.5 & $0.0233 \pm 0.0050$ & 13.45 & 1 & - & 1.0 \\
\hline 28 & Mkn 704 & 1RXS J091826.2+161825 & 0.029 & 2.5 & 1.1 & 5.5 & $0.0274 \pm 0.0061$ & 14.12 & - & 1 & 1.0 \\
\hline 29 & Mkn 110 & 1RXP J092512.8+521712 & 0.036 & 1.2 & - & - & - & 15.37 & 1 & 1 & 1.0 \\
\hline 30 & Mkn 705 & 1RXP J092603.0+124359 & 0.028 & 2.1 & - & - & - & 14.55 & 1 & 1 & $1.0^{\#}$ \\
\hline 31 & NGC 2992 & 1RXP J094541.6-141938 & 0.007 & 5.6 & 2.7 & 13.5 & $0.0239 \pm 0.0051$ & 13.78 & 1 & 1 & 1.9 \\
\hline 32 & Mkn 124 & 1RXS J094841.6+502926 & 0.056 & 1.0 & - & - & - & 15.33 & - & 1 & 1.0 \\
\hline 33 & Mkn 1239 & 1RXP J095219.0-013631 & 0.019 & 1.3 & - & - & - & 14.33 & 1 & 1 & $1.5^{\#}$ \\
\hline 34 & NGC 3031 & 1RXP J095532.8+690354 & 0.000 & 6.9 & - & - & - & 11.72 & 1 & 1 & 1.5 \\
\hline 35 & NGC 3080 & 1RXP J095956.3+130242 & 0.035 & 2.2 & - & - & - & 15.01 & 1 & 1 & 1.0 \\
\hline 36 & NGC 3185 & 1RXP J101737.8+214124 & 0.004 & 0.7 & - & - & - & 12.73 & 1 & - & 1.0 \\
\hline 37 & Mkn 141 & 1RXS J101912.1+635802 & 0.039 & 1.5 & 1.4 & 2.8 & $0.1386 \pm 0.0349$ & 15.27 & 1 & 1 & 1.2 \\
\hline 38 & NGC 3227 & 1RXP J102330.2+195150 & 0.003 & 12.5 & 8.0 & 11.0 & $0.7513 \pm 0.1615$ & 11.29 & 1 & 1 & 1.0 \\
\hline 39 & Mkn 142 & 1RXP J102532.5+514045 & 0.045 & 1.0 & - & - & - & 15.50 & 1 & 1 & $1.0^{\#}$ \\
\hline 40 & Mkn 634 & 1RXS J105801.2+202937 & 0.066 & 1.8 & - & - & - & 15.49 & - & 2 & 1.0 \\
\hline 41 & NGC 3516 & 1RXP J110649.0+723406 & 0.009 & 7.0 & 1.7 & 21.0 & $0.0044 \pm 0.0009$ & 12.23 & 1 & 1 & 1.0 \\
\hline 42 & Mkn 732 & 1RXS J111349.5+093518 & 0.030 & 2.6 & - & - & - & 14.00 & - & 1 & 1.0 \\
\hline 43 & Mkn 734 & 1RXP J112146.9+114418 & 0.049 & 1.1 & - & - & - & 14.71 & 1 & 1 & $1.0^{\#}$ \\
\hline 44 & Mkn 40 & 1RXP J112536.6+542303 & 0.020 & 1.3 & 0.7 & 1.5 & $0.2572 \pm 0.0851$ & 15.39 & 1 & 2 & 1.0 \\
\hline 45 & Mkn 739A & 1RXP J113629.2+213543 & 0.030 & 1.8 & - & - & - & 14.02 & 1 & 1 & 1.0 \\
\hline 46 & Mkn 744 & 1RXP J113942.8+315439 & 0.010 & 4.8 & 7.5 & 6.8 & $0.6870 \pm 0.1507$ & 13.74 & 1 & 1 & 1.8 \\
\hline 47 & NGC 3884 & 1RXS J114611.5+202355 & 0.023 & 6.7 & - & - & - & 12.40 & - & 1 & 1.0 \\
\hline 48 & Mkn 42 & 1RXP J115341.7+461254 & 0.024 & 2.1 & - & - & - & 15.45 & 1 & 1 & $1.0^{\#}$ \\
\hline 49 & Mkn 1310 & 1RXP J120114.9-034031 & 0.019 & 1.2 & - & - & - & 15.08 & 1 & 1 & 1.0 \\
\hline 50 & NGC 4051 & 1RXP J120310.2+443156 & 0.002 & 19.0 & - & - & - & 12.12 & 1 & 1 & $1.0^{\#}$ \\
\hline 51 & NGC 4151 & 1RXP J121032.4+392418 & 0.003 & 12.5 & 3.6 & 23.5 & $0.0233 \pm 0.0049$ & 11.26 & 1 & 1 & 1.0 \\
\hline 52 & Mkn 1469 & 1RXS J121607.4+504926 & 0.031 & 1.9 & - & - & - & 14.20 & - & 1 & 1.5 \\
\hline 53 & NGC 4235 & 1RXP J121710.1+071135 & 0.007 & 5.1 & - & - & - & 13.50 & 1 & 1 & 1.0 \\
\hline 54 & Mkn 766 & 1RXP J121826.4+294847 & 0.012 & 3.0 & - & - & - & 13.00 & 1 & 1 & $1.0^{\#}$ \\
\hline 55 & NGC 4258 & 1RXP J121856.4+471755 & 0.002 & 37.0 & 3.0 & 12.0 & $0.6768 \pm 0.1452$ & 11.30 & 1 & 1 & 1.0 \\
\hline 56 & NGC 4278 & 1RXP J122007.2+291646 & 0.002 & 7.5 & 3.3 & 12.0 & $0.0713 \pm 0.0153$ & 10.51 & 1 & 1 & 1.0 \\
\hline 57 & Mkn 205 & 1RXP J122144.5+751840 & 0.070 & 1.1 & 6.0 & 3.3 & $0.4718 \pm 0.1139$ & 15.24 & 1 & 1 & 1.0 \\
\hline 58 & Mkn 50 & 1RXS J122324.4+024040 & 0.023 & 1.0 & - & - & - & 15.17 & - & 2 & 1.0 \\
\hline 59 & NGC 4593 & 1RXP J123939.2-052046 & 0.009 & 17.0 & 3.0 & 18.0 & $0.0625 \pm 0.0133$ & 13.19 & 1 & 1 & 1.0 \\
\hline 60 & NGC 4594 & 1RXP J123959.2-113731 & 0.002 & 33.0 & - & - & - & 9.64 & 1 & 1 & 1.0 \\
\hline 61 & NGC 4639 & 1RXP J124252.2+131527 & 0.001 & 6.0 & - & - & - & 11.00 & 1 & 1 & 1.0 \\
\hline 62 & IR $1249-1308$ & 1RXS J125212.5-132450 & 0.014 & 2.7 & - & - & - & 14.47 & - & 1 & $1.0^{\#}$ \\
\hline 63 & Mkn 236 & 1RXS J130021.2+613919 & 0.052 & 1.5 & - & - & - & 15.45 & - & 1 & 1.0 \\
\hline 64 & Mkn 783 & 1RXS J130258.8+162423 & 0.067 & 0.7 & - & - & - & 15.50 & - & 1 & $1.0^{\#}$ \\
\hline 65 & NGC 5033 & 1RXP J131327.7+363536 & 0.003 & 41.0 & - & - & - & 12.37 & 1 & 1 & 1.0 \\
\hline 66 & Mkn 1347 & 1RXS J132254.2+081011 & 0.050 & 1.9 & - & - & - & 14.38 & - & 2 & 1.0 \\
\hline 67 & NGC 5273 & 1RXP J134208.3+353919 & 0.003 & 10.0 & 3.6 & 15.5 & $0.0580 \pm 0.0124$ & 13.44 & 1 & 2 & 1.0 \\
\hline 68 & Mkn 279 & 1RXH J135303.5+691830 & 0.031 & 2.7 & 1.3 & 3.6 & $0.1409 \pm 0.0334$ & 14.45 & 1 & 1 & 1.0 \\
\hline 69 & Mkn 662 & 1RXS J135405.7+232549 & 0.055 & 1.0 & - & - & - & 15.24 & - & 2 & 1.5 \\
\hline 70 & NGC 5548 & 1RXP J141759.3+250811 & 0.017 & 5.0 & - & - & - & 13.46 & 1 & 1 & 1.5 \\
\hline 71 & Mkn 684 & 1RXS J143104.8+281716 & 0.046 & 4.0 & 2.0 & 9.0 & $0.0310 \pm 0.0067$ & 14.68 & 1 & 1 & $2.0^{\#}$ \\
\hline 72 & Mkn 471 & 1RXP J142255.5+325111 & 0.034 & 3.6 & - & - & - & 14.42 & 1 & 1 & 1.9 \\
\hline
\end{tabular}


Table C.1. continued

\begin{tabular}{|c|c|c|c|c|c|c|c|c|c|c|c|}
\hline Nr. & name & ROSAT name & $z$ & $\begin{array}{c}D_{\mathrm{p}} \\
\mathrm{mm}\end{array}$ & $\begin{array}{c}D_{\mathrm{c}} \\
\mathrm{mm}\end{array}$ & $\begin{array}{c}S \\
\mathrm{~mm}\end{array}$ & $\overline{\bar{Q}}$ & $\begin{array}{c} \\
\mathrm{mag}\end{array}$ & $\begin{array}{l}\mathrm{i} \\
\text { point. }\end{array}$ & sur. & $\begin{array}{c}\text { Sy } \\
\text { type }\end{array}$ \\
\hline 73 & Mkn 474 & 1RXP J143452.1+483933 & 0.041 & 1.2 & $\overline{-}$ & $\overline{-}$ & - & $\overline{15.18}$ & $\overline{11}$ & $\overline{11}$ & 1.0 \\
\hline 74 & Mkn 817 & 1RXP J143622.9+584737 & 0.033 & 2.1 & - & - & - & 13.79 & 1 & 1 & 1.0 \\
\hline 75 & Mkn 1494 & 1RXP J150139.6+102521 & 0.031 & 2.5 & 0.7 & 7.1 & $0.0065 \pm 0.0014$ & 15.50 & 1 & - & 1.0 \\
\hline 76 & Mkn 841 & 1RXP J150401.4+102617 & 0.036 & 1.2 & - & - & - & 14.92 & 1 & 1 & 1.0 \\
\hline 77 & Mkn 1392 & 1RXS J150556.3+034212 & 0.036 & 3.1 & - & - & - & 14.14 & - & 1 & 1.5 \\
\hline 78 & Mkn 845 & 1RXS J150744.6+512709 & 0.042 & 2.8 & - & - & - & 14.21 & - & 1 & 1.0 \\
\hline 79 & IR $1509-2107$ & 1RXP J151159.6-211903 & 0.044 & 0.7 & - & - & - & 14.04 & 1 & 1 & $1.0^{\#}$ \\
\hline 80 & NGC 5940 & 1RXS J153118.2+072713 & 0.033 & 2.6 & - & - & - & 14.20 & - & 1 & 1.0 \\
\hline 81 & Mkn 290 & 1RXP J153552.8+575411 & 0.029 & 1.5 & - & - & - & 14.62 & 1 & 1 & 1.0 \\
\hline 82 & Mkn 486 & 1RXP J153638.0+543336 & 0.039 & 1.0 & - & - & - & 14.68 & 1 & 2 & 1.0 \\
\hline 83 & Mkn 291 & 1RXP J155507.6+191139 & 0.035 & 1.4 & - & - & - & 15.50 & 2 & 1 & $1.0^{\#}$ \\
\hline 84 & Mkn 493 & 1RXP J155909.7+350154 & 0.031 & 3.1 & - & - & - & 15.06 & 1 & 1 & $1.5^{\#}$ \\
\hline 85 & NGC 6104 & 1RXS J161630.6+354204 & 0.028 & 6.0 & 2.0 & 16.7 & $0.0089 \pm 0.0019$ & 14.00 & - & 2 & 1.0 \\
\hline 86 & Mkn 699 & 1RXP J162347.0+410433 & 0.034 & 0.9 & - & - & - & 15.19 & 2 & 2 & 1.2 \\
\hline 87 & Mkn 885 & 1RXS J162948.3+672247 & 0.026 & 2.6 & - & - & - & 14.17 & - & 1 & 1.0 \\
\hline 88 & Mkn 883 & 1RXP J162953.3+242640 & 0.038 & 1.2 & - & - & - & 14.43 & 1 & 1 & 1.9 \\
\hline 89 & NGC 6212 & 1RXP J164322.5+394823 & 0.030 & 1.9 & - & - & - & 15.00 & 1 & - & 1.0 \\
\hline 90 & NGC 6240 & 1RXP J165259.0+022406 & 0.024 & 5.0 & - & - & - & 13.37 & 1 & 1 & 3.0 \\
\hline 91 & Mkn 506 & 1RXP J172239.8+305245 & 0.043 & 2.3 & 1.7 & 3.7 & $0.1526 \pm 0.0360$ & 14.55 & 1 & 1 & 1.5 \\
\hline 92 & NGC 6814 & 1RXP J194240.7-101928 & 0.005 & 7.8 & - & - & - & 14.37 & 1 & 1 & 1.0 \\
\hline 93 & Mkn 896 & 1RXP J204620.8-024848 & 0.027 & 2.3 & 0.7 & 6.0 & $0.0095 \pm 0.0021$ & 14.28 & 1 & 1 & $1.0^{\#}$ \\
\hline 94 & Mkn 516 & 1RXP J215622.2+072213 & 0.028 & 1.7 & 1.0 & 3.6 & $0.0475 \pm 0.0113$ & 15.01 & 1 & - & 1.8 \\
\hline 95 & Mkn 915 & 1RXS J223647.3-123228 & 0.025 & 3.4 & 2.1 & 9.8 & $0.0203 \pm 0.0044$ & 14.03 & - & 2 & 1.5 \\
\hline 96 & IR $2237+0747$ & 1RXH J224017.3+080314 & 0.025 & 3.5 & - & - & - & 14.00 & 1 & 1 & 1.0 \\
\hline 97 & Mkn 1126 & 1RXS J230048.1-125518 & 0.010 & 2.5 & - & - & - & 14.11 & - & 1 & $1.0^{\#}$ \\
\hline 98 & NGC 7469 & 1RXP J230315.6+085233 & 0.017 & 3.0 & 2.7 & 6.3 & $0.0922 \pm 0.0203$ & 13.72 & 1 & 1 & 1.0 \\
\hline 99 & Mkn 315 & 1RXS J230402.8+223725 & 0.040 & 1.4 & 0.8 & 2.4 & $0.0857 \pm 0.0227$ & 14.09 & 1 & 1 & 1.0 \\
\hline 100 & NGC 7603 & 1RXP J231856.6+001448 & 0.029 & 4.2 & 0.8 & 4.8 & $0.0557 \pm 0.0126$ & 14.21 & 1 & 1 & 1.0 \\
\hline 101 & Mkn 541 & 1RXS J235602.1+073121 & 0.040 & 1.2 & - & - & - & 15.15 & - & 2 & 1.0 \\
\hline 102 & Mkn 543 & 1RXS J000226.6+032105 & 0.026 & 2.0 & - & - & - & 14.09 & - & 1 & 1.0 \\
\hline
\end{tabular}

Table C.2. Seyfert 2 galaxies: A part of the Rafanelli sample of interacting or isolated Seyfert 2 galaxies detected by ROSAT. The table contains optical and generical properties as well as some results from the investigations of Rafanelli et al. (1995). For the description of columns see Table C.1

\begin{tabular}{|c|c|c|c|c|c|c|c|c|c|c|c|}
\hline Nr. & name & ROSAT name & $\bar{z}$ & $\begin{array}{c}D_{\mathrm{p}} \\
\mathrm{mm} \\
\end{array}$ & $\begin{array}{c}D_{\mathrm{c}} \\
\mathrm{mm}\end{array}$ & $\begin{array}{c}S \\
\mathrm{~mm} \\
\end{array}$ & $\overline{Q Q}$ & $\begin{array}{c}V \\
\mathrm{mag}\end{array}$ & $\begin{array}{l}\text { i } \\
\text { point. }\end{array}$ & sur. & $\begin{array}{c}\text { Sy } \\
\text { type }\end{array}$ \\
\hline$\overline{\overline{1}}$ & Mkn 348 & 1RXP J004847.4+315716 & $\overline{0.014}$ & 2.0 & $\overline{0.5}$ & $\overline{\overline{5.0}}$ & $\overline{0.0080 \pm 0.0018}$ & 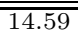 & $\overline{\overline{1}}$ & $\overline{-}$ & $\overline{2.0}$ \\
\hline 2 & IR $0135-1307$ & 1RXP J013805.4-125213 & 0.041 & 1.6 & - & - & - & 15.30 & 1 & - & 2.0 \\
\hline 3 & Mkn 573 & 1RXP J014357.8+022050 & 0.017 & 2.3 & - & - & - & 14.07 & 1 & 1 & 2.0 \\
\hline 4 & IR $0147-0740$ & 1RXP J015002.7-072540 & 0.017 & 1.0 & - & - & - & 15.62 & 1 & - & 2.0 \\
\hline 5 & NGC 1068 & 1RXP J024240.9-000042 & 0.003 & 28.0 & - & - & - & 10.83 & 1 & 1 & 2.0 \\
\hline 6 & NGC 1144 & 1RXP J025511.9-001042 & 0.029 & 3.2 & 1.5 & 2.4 & $0.7607 \pm 0.2015$ & 14.41 & 2 & - & 2.0 \\
\hline 7 & IR0 253-1641 & 1RXS J025601.7-162919 & 0.033 & 1.7 & - & - & - & 15.50 & - & 1 & 2.0 \\
\hline 8 & Mkn 1066 & & 0.012 & 4.2 & - & - & - & 13.96 & 1 & - & 2.0 \\
\hline 9 & Mkn 607 & 1RXS J032446.8-030256 & 0.009 & 2.5 & 6.0 & 7.5 & $0.1377 \pm 0.0300$ & 14.00 & - & 1 & 2.0 \\
\hline 10 & IR0 $450-0317$ & 1WGA J0452.7-0312 & 0.016 & 2.0 & - & - & - & 15.00 & 2 & - & 2.0 \\
\hline 11 & NGC 2110 & 1RXH J055211.4-072725 & 0.007 & 2.5 & - & - & - & 13.51 & 1 & 1 & 2.0 \\
\hline 12 & Mkn 3 & 1RXP J061535.6+710209 & 0.014 & 3.5 & - & - & - & 13.34 & 1 & 2 & 2.0 \\
\hline 13 & Mkn 620 & & 0.006 & 5.0 & - & - & - & 13.54 & 1 & - & 2.0 \\
\hline 14 & Mkn 78 & 1RXP J074241.2+651031 & 0.038 & 1.6 & - & - & - & 14.58 & 1 & - & 2.0 \\
\hline 15 & Mkn 1210 & 1RXS J080404.6+050641 & 0.013 & 2.2 & - & - & - & 15.00 & - & 2 & 2.0 \\
\hline 16 & NGC 3081 & 1RXS J095930.3-224954 & 0.007 & 6.1 & 1.3 & 8.8 & $0.0328 \pm 0.0071$ & 13.55 & - & 2 & 2.0 \\
\hline 17 & Mkn 720 & 1RXS J101737.6+065820 & 0.045 & 1.6 & - & - & - & 15.27 & - & 1 & 2.0 \\
\hline 18 & Mkn 34 & & 0.051 & 1.4 & - & - & - & 14.65 & 1 & - & 2.0 \\
\hline 19 & NGC 3660 & 1RXS J112332.4-083932 & 0.011 & 6.5 & - & - & - & 14.45 & - & 1 & 2.0 \\
\hline 20 & NGC 3982 & 1RXP J115628.0+550731 & 0.003 & 6.9 & - & - & - & 11.70 & 1 & - & 2.0 \\
\hline 21 & NGC 4388 & 1RXP J122546.7+123946 & 0.008 & 16.0 & 2.3 & 39.0 & $0.0038 \pm 0.0008$ & 13.90 & 1 & - & 2.0 \\
\hline 22 & NGC 4922B & & 0.024 & 4.3 & - & - & - & 15.00 & 1 & - & 2.0 \\
\hline 23 & NGC 4941 & 1RXS J130413.2-053304 & 0.003 & 11.0 & - & - & - & 12.23 & - & 1 & 2.0 \\
\hline 24 & NGC 5005 & 1RXP J131056.3+370323 & 0.003 & 23.0 & - & - & - & 13.67 & 1 & 1 & 2.0 \\
\hline 25 & IR $1329+0216$ & 1RXP J133152.2+020057 & 0.086 & 1.0 & - & - & - & 15.00 & 2 & 1 & 2.0 \\
\hline 26 & NGC 5252 & & 0.022 & 4.2 & 0.7 & 8.9 & $0.0072 \pm 0.0015$ & 14.21 & 1 & - & 2.0 \\
\hline 27 & Mkn 266SW & 1RXP J133818.7+481641 & 0.028 & 2.5 & - & - & - & 13.42 & 1 & 1 & 2.0 \\
\hline 28 & NGC 5506 & 1RXP J141315.0-031218 & 0.007 & 8.9 & 3.5 & 12.5 & $0.0890 \pm 0.0191$ & 14.38 & 1 & 1 & 2.0 \\
\hline 29 & Mkn 670 & 1RXS J141417.5+264441 & 0.035 & 1.5 & - & - & - & 14.68 & 2 & 2 & 2.0 \\
\hline 30 & Mkn 673 & 1RXP J141721.4+265141 & 0.036 & 4.5 & - & - & - & 15.00 & 1 & - & 2.0 \\
\hline 31 & NGC 5929 & 1RXP J152607.0+414016 & 0.008 & 5.2 & - & - & - & 14.00 & 1 & - & 2.0 \\
\hline 32 & NGC 5953 & 1RXH J153432.8+151137 & 0.007 & 4.7 & 5.5 & 3.5 & $3.0654 \pm 0.7308$ & 13.10 & 1 & 1 & 2.0 \\
\hline 33 & NGC 6211 & 1RXS J164118.4+574601 & 0.020 & 6.5 & 2.5 & 10.0 & $0.0655 \pm 0.0141$ & 14.30 & - & 2 & 2.0 \\
\hline 34 & NGC 7319 & 1RXH J223603.2+335833 & 0.022 & 3.5 & 4.8 & 6.2 & $0.2889 \pm 0.0638$ & 13.53 & 1 & 2 & 2.0 \\
\hline 35 & NGC 7674 & 1RXP J232757.0+084644 & 0.029 & 4.0 & 2.0 & 11.0 & $0.0170 \pm 0.0037$ & 14.36 & 1 & - & 2.0 \\
\hline 36 & NGC 7743 & & 0.007 & 8.0 & - & - & - & 13.28 & 2 & - & 2.0 \\
\hline
\end{tabular}


Table C.3. Seyfert 1 galaxies: The table contains soft X-ray properties of the interacting or isolated Seyfert 1 galaxies. Col. (1) - object name, Cols. (2 and 3$)-R O S A T$ position (pointed observations are preferred), Cols. (4 and 5) - pointing ( $p=$ PSPC, $h=$ HRI detector), survey (RASS II catalogue) count rates, Cols. (6 and 7 ) - exposure times, Cols. (8 and 9$)$ - logarithmic fluxes $(f=$ from fit, $c=$ from count rate), Cols. (10 and 11) - logarithmic luminosities, Cols. (12 and 13$)-$ galactic and spectral hydrogen column densities (三 fixed value for $N_{\mathrm{H}_{\mathrm{fit}}}<N_{\mathrm{H}_{\mathrm{gal}}}$ or no spectral $\Gamma$ ), Col.(14) - monochromatic flux at $1 \mathrm{keV}$ and Col. (15) - photon index. In Cols. (2), (3), (13), (14) and (15) we have preferred the data from the pointed spectral fit opposed to the survey spectral fit. Note that PSPC and HRI count rates are not comparable and see the discussion about column densities of Seyfert 2 in Sect. 4

\begin{tabular}{|c|c|c|c|c|c|c|c|c|c|c|c|c|c|c|}
\hline \multirow[t]{3}{*}{ name } & \multicolumn{2}{|c|}{ ROSAT position } & \multirow{2}{*}{\multicolumn{2}{|c|}{$\begin{array}{c}\text { count rate } \\
{\left[\text { counts s}^{-1}\right]}\end{array}$}} & \multirow{2}{*}{\multicolumn{2}{|c|}{$\begin{array}{c}\begin{array}{c}\text { expo } \\
{[\mathrm{s}]}\end{array} \\
\end{array}$}} & \multirow{2}{*}{\multicolumn{2}{|c|}{$\begin{array}{c}\log f_{\mathrm{X}} \\
{\left[\mathrm{erg} \mathrm{cm}^{-2} \mathrm{~s}^{-1}\right]}\end{array}$}} & \multirow{2}{*}{\multicolumn{2}{|c|}{$\begin{array}{c}\log L_{X} \\
{\left[\operatorname{erg~s}^{-1}\right]}\end{array}$}} & \multirow{2}{*}{\multicolumn{2}{|c|}{$\begin{array}{c}N_{\mathrm{H}} \\
{\left[10^{21} \mathrm{~cm}^{-2}\right]}\end{array}$}} & \multirow{3}{*}{$\begin{array}{c}f_{1 \mathrm{keV}} \\
{\left[10^{-5} \mathrm{phot} \mathrm{s}^{-1}\right.} \\
\left.\mathrm{cm}^{-2} \mathrm{keV}^{-1}\right]\end{array}$} & \\
\hline & & $\delta_{2000}$ & & & & & & & & & & & & \\
\hline & {$[\mathrm{h}][\mathrm{m}][\mathrm{s}$} & {$\left[^{\circ}\right]\left[\left[^{\prime}\right][\right.$} & inting & survey & nt. & 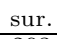 & point & sur. & oint. & sur. & gal & & & \\
\hline Mkn 334 & 000308.6 & 215728 & - & $0.096 \pm 0.020$ & & 293 & - & $-11.59^{c}$ & & 42.38 & 0.443 & & & $\equiv-2.30$ \\
\hline Mkn 335 & 0619.2 & 201225 & $\pm 0.011^{p}$ & $2.482 \pm 0.097$ & 24337 & 269 & $-9.95^{f}$ & $-9.81^{f}$ & 44.14 & 44.28 & 0.396 & $0.396 \pm 0.016$ & $682.74 \pm \varepsilon$ & $-3.04 \pm 0.01$ \\
\hline Mkn 1146 & 4719.4 & $1442 \quad 15$ & +0 & $0.067 \pm 0.015$ & 等 & 370 & - & $-11.74^{c}$ & Nat & 42.73 & 0.460 & 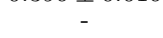 & & $\equiv-2.30$ \\
\hline Mkn 352 & 05953.3 & 314948 & $0.093 \pm 0.005^{p}$ & $0.615 \pm 0.037$ & 4418 & 462 & $-11.63^{f}$ & $-10.73^{f}$ & 42.01 & 42.91 & 0.549 & $0.651 \pm 0.136$ & $47.65 \pm 15.86$ & $-1.94 \pm 0.43$ \\
\hline Mkn 1152 & 1350.1 & -145034 & $923 \pm 0.024^{p}$ & $0.946 \pm 0.050$ & 3219 & 412 & $-10.49^{f}$ & -10 & 44.24 & 43.74 & .163 & $0.209 \pm 0.026$ & $421.55 \pm$ & $-2.45 \pm 0.10$ \\
\hline Mkn & 2531.4 & 320800 & - & $0.083 \pm 0.017$ & & 328 & - & $-11.59^{c}$ & 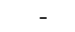 & 42.15 & 0.571 & - & - & $\equiv-2.30$ \\
\hline Mkn S & 1350.4 & 131534 & - & $0.030 \pm 0.012$ & - & 462 & - & $-12.14^{c}$ & - & 42.56 & 0.390 & - & - & $\equiv-2.30$ \\
\hline Mkn 358 & 2634.2 & 313659 & - & $0.172 \pm 0.026$ & & 303 & - & $-10.89^{f}$ & 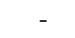 & 43.66 & 0.602 & $\equiv 0.602$ & $69.30 \pm 19.90$ & $-3.12 \pm 0.39$ \\
\hline Mkn 359 & 2732.8 & 191051 & $822 \pm 0.016^{p}$ & $0.608 \pm 0.042$ & 3187 & 388 & $-10.53^{f}$ & $-10.37^{f}$ & 43.22 & 43.38 & 0.483 & $0.543 \pm 0.068$ & $356.61 \pm$ & $-2.51 \pm 0.13$ \\
\hline Mkn 1018 & 0616.0 & $-00 \quad 1732$ & $.239 \pm 0.006^{p}$ & $0.081 \pm 0.017$ & 6510 & 395 & $-11.26^{f}$ & $-11.83^{c}$ & 43.30 & 42.73 & 0.255 & $0.342 \pm 0.073$ & $81.50 \pm 5.20$ & $-2.33 \pm 0.18$ \\
\hline Mkn 590 & 1433.7 & -004604 & $.225 \pm 0.039^{p}$ & $2.689 \pm 0.167$ & 3484 & 311 & $-9.89^{f}$ & $-10.30^{f}$ & 44.27 & 43.85 & 0.272 & $0.317 \pm 0.020$ & $1459.10 \pm 29.21$ & $-2.58 \pm 0.05$ \\
\hline Mkn 1040 & 2814.6 & 311838 & - & $0.342 \pm 0.033$ & 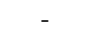 & 3 & - & $-11.20^{f}$ & 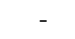 & 42.48 & 0.674 & $\equiv 0.674$ & $160.00 \pm 30.80$ & $-0.83 \pm 0.64$ \\
\hline Mkn 1044 & 3005.8 & -085940 & $.119 \pm 0.027^{p}$ & $2.141 \pm 0.128$ & 2836 & 278 & $-10.02^{f}$ & $-10.12^{f}$ & 43.68 & 43.58 & 0.316 & $0.421 \pm 0.040$ & $549.43 \pm 1$ & $-3.08 \pm 0.09$ \\
\hline & 3437.9 & -084709 & $331 \pm 0.015^{p}$ & $1.281 \pm$ & 6191 & & $-10.43^{f}$ & -10 & 44.14 & 44.16 & & $0.342 \pm$ & $360.98 \pm$ & $-2.69 \pm 0.08$ \\
\hline Mkn & 4135.2 & $0711 \quad 17$ & -5 & $0.147 \pm 0.029$ & & 198 & 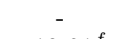 & -11 & & 42.88 & 0.676 & & & $\equiv-2.30$ \\
\hline Mkn 3 & 4920.6 & 191822 & $282 \pm 0.005^{p}$ & $0.234 \pm 0.026$ & 12675 & 368 & $-10.91^{f}$ & $-10.76^{f}$ & 43.36 & 43.52 & 0.962 & $\equiv 0.962$ & $173.41 \pm 4.63$ & $-2.37 \pm 0.07$ \\
\hline & 2525.5 & -060827 & $\pm 0.006^{p}$ & $.413 \pm$ & 5801 & & $-11.27^{f}$ & -10 & 43.03 & 43.50 & & $0.585=$ & $93.81=$ & $-2.14 \pm 0.26$ \\
\hline M & 3622.1 & -102231 & $\pm 0.010^{h}$ & $0.579 \pm$ & 3001 & 370 & $10.41^{c}$ & -10 & 43.97 & 43.69 & 0.583 & $\equiv 0.5$ & $210.00 \pm$ & $-2.64 \pm 0.27$ \\
\hline & 5209.8 & 742537 & & $0.062 \pm 0$ & & 4 & 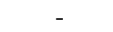 & $-11.69^{c}$ & & 42.15 & 0.646 & - & & $\equiv-2.30$ \\
\hline & $59:$ & 541 & 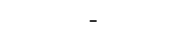 & & - & & - & & - & 44.31 & & & 24900 & -3.20 \\
\hline & 36 & 584 & - & 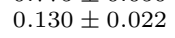 & & & - & -1 & - & 43.03 & & & & $=-2.30$ \\
\hline Mkn & 4233.5 & 494838 & $2 \pm 0.026^{p}$ & $196 \pm 0$. & 2690 & 3 & $-9.99^{f}$ & $-9.94^{f}$ & 43.99 & 44.03 & 0.566 & $790 \pm 0.06$ & $1039.90 \pm$ & $-2.66 \pm 0.10$ \\
\hline & 4728.6 & 605553 & $1 \pm 0.015^{p}$ & - & 4246 & & $-10.49^{f}$ & $-10.69^{f}$ & 43.75 & 43.56 & & $\equiv 0.482$ & $390.60 \pm$ & $-2.51 \pm 0.04$ \\
\hline $\mathrm{Mk}$ & 5525.1 & 391113 & $2 \pm 0.006^{h}$ & $449 \pm 0$. & 3535 & 42 & $10.65^{c}$ & -10 & 43.71 & 43.78 & 0.532 & $\equiv 0.5$ & $146.00 \pm 2$ & $-3.09 \pm 0.23$ \\
\hline & 3810.8 & 245337 & $3 \pm 0.013^{p}$ & $213 \pm 0$ & 2879 & 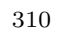 & $-11.02^{f}$ & $-11.31^{c}$ & 43.15 & 42.87 & & $0.449 \pm \mathrm{c}$ & $235.21 \pm$ & $-1.50 \pm 0.22$ \\
\hline & 43 & 5012 & c & 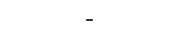 & 8749 & 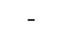 & $-12.41^{f}$ & 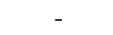 & & 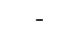 & & $\equiv 0.3$ & $6.62 \pm 1$ & $-2.16 \pm 0.24$ \\
\hline $\mathrm{NC}$ & 1405.0 & 400648 & $1 \pm 0$. & - & 21715 & - & $-12.19^{c}$ & - & 40.90 & & 80 & $-\infty$ & & $\equiv-2.30$ \\
\hline & 1826.2 & 161825 & & $0.756 \pm 0$ & - & . & the & -10 & - & 43.61 & & $0.407 \pm$ & $219.00 \pm$ & $-2.76 \pm 0.52$ \\
\hline & 25 & 5217 & +5 & 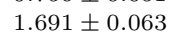 & 6013 & & $-9.97^{f}$ & -10 & 44.44 & & & $0.193 \pm$ & $1181.2 \pm$ & $-2.59 \pm 0.04$ \\
\hline M & 2603 & 4400 & $1 \pm 0$ & $6 \pm$ & 5244 & & $-10.54^{f}$ & -10 & 43.65 & 43 & & $0.398 \pm$ & $342.95=$ & $-2.53 \pm 0.09$ \\
\hline & 45 & -1 & \pm 0 & & 10 & & $-11.41^{f}$ & & 41.56 & & & $2.101 \pm$ & $72.41 \pm$ & 36 \\
\hline & 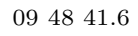 & & - & & & & 1. & & - & & & - & - & -2 \\
\hline & $521 !$ & -013632 & \pm 0 & $4 \pm$ & 43 & & $10.69^{f}$ & -11 & 43.1 & 41 & & $0.859 \pm \mathrm{c}$ & $22.42 \pm$ & $-4.06 \pm 0.30$ \\
\hline & 553 & 690354 & 10 & $8 \pm$ & & & & & & 40 & & $12 \pm$ & 396.29 & $28 \pm 0.07$ \\
\hline & 595 & 130243 & \pm 0 & $0.219 \pm 0$ & 1080 & 255 & $1.10^{f}$ & $-11.32^{c}$ & 43.28 & 43.06 & & $0.367 \pm$ & $92.95 \pm$ & $-2.54 \pm 0.39$ \\
\hline & 17 & & & - & & - & $-12.95^{f}$ & - & 39.53 & & & $0.279 \pm$ & & $.47 \pm 2.64$ \\
\hline & 19 & 635802 & \pm 0 & $6 \pm 0$ & & 498 & $3^{c}$ & -11 & 42.75 & 43 & 7 & $0.146 \pm$ & $82.00 \pm$ & $-2.56 \pm 0.62$ \\
\hline & 10233 & 195150 & $60 \pm 0.0$ & 0 & 19547 & & $1.00^{f}$ & -11 & 41.2 & & & $0.396 \pm$ & $259.21 \pm$ & $-1.25 \pm 0.08$ \\
\hline & & & $1.373 \pm 0.0$ & & 8427 & & $-10.41^{f}$ & & 44.21 & & & $0.252 \pm($ & $163.55 \pm$ & \\
\hline & 580 & 37 & & $4 \pm 0$ & & & & & & 43 & 186 & & & -2.30 \\
\hline NC & 064 & 723407 & $4.711 \pm 0.019^{p}$ & & 13079 & & $-9.93^{f}$ & -11 & 43.26 & & & $\equiv 0.3$ & 1595.40 & $-2.40 \pm 0.01$ \\
\hline & 134 & . & & & & & - & & & & & $438 \pm$ & $98.30 \pm$ & $64 \pm 0.96$ \\
\hline Mkn 7 & 2146.9 & 114418 & $50 \pm 0$. & $418 \pm 0$ & 4011 & 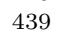 & $-10.48^{f}$ & -11 & 44.22 & 43.67 & 0.264 & $0.450 \pm 0$ & $77.07 \pm 6$ & $-3.63 \pm 0.19$ \\
\hline & 253 & & ( & & & & $i^{f}$ & & & & & + & .72 & 12 \\
\hline & 362 & 3543 & 50 & & & & & & 43 & & & $32 \pm c$ & $77 \pm$ & $3 \pm 0.14$ \\
\hline & 39 & & $1 \pm 0.0$ & & 2866 & & $-11.10^{f}$ & & 42.18 & 40 & & $0.652 \pm 0$ & $06.54 \pm$ & \pm 0.18 \\
\hline & 46 & & 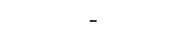 & & - & & ( & & 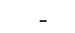 & & & - & - & 2.30 \\
\hline Mkn 42 & 5341.7 & 461255 & \pm 0 & $.192 \pm 0$ & 44 & & -1 & & 42.81 & & & $0.283 \pm$ & $8 \pm$ & $-2.76 \pm 0.23$ \\
\hline Mkn 1310 & 120114.9 & -034031 & $0.188 \pm 0.004^{p}$ & $0.831 \pm 0.073$ & 13559 & 339 & $-11.43^{f}$ & $-10.82^{f}$ & 42.41 & 43.02 & 0.243 & $0.303 \pm 0.056$ & $64.60 \pm 3.38$ & $-2.13 \pm 0.16$ \\
\hline
\end{tabular}




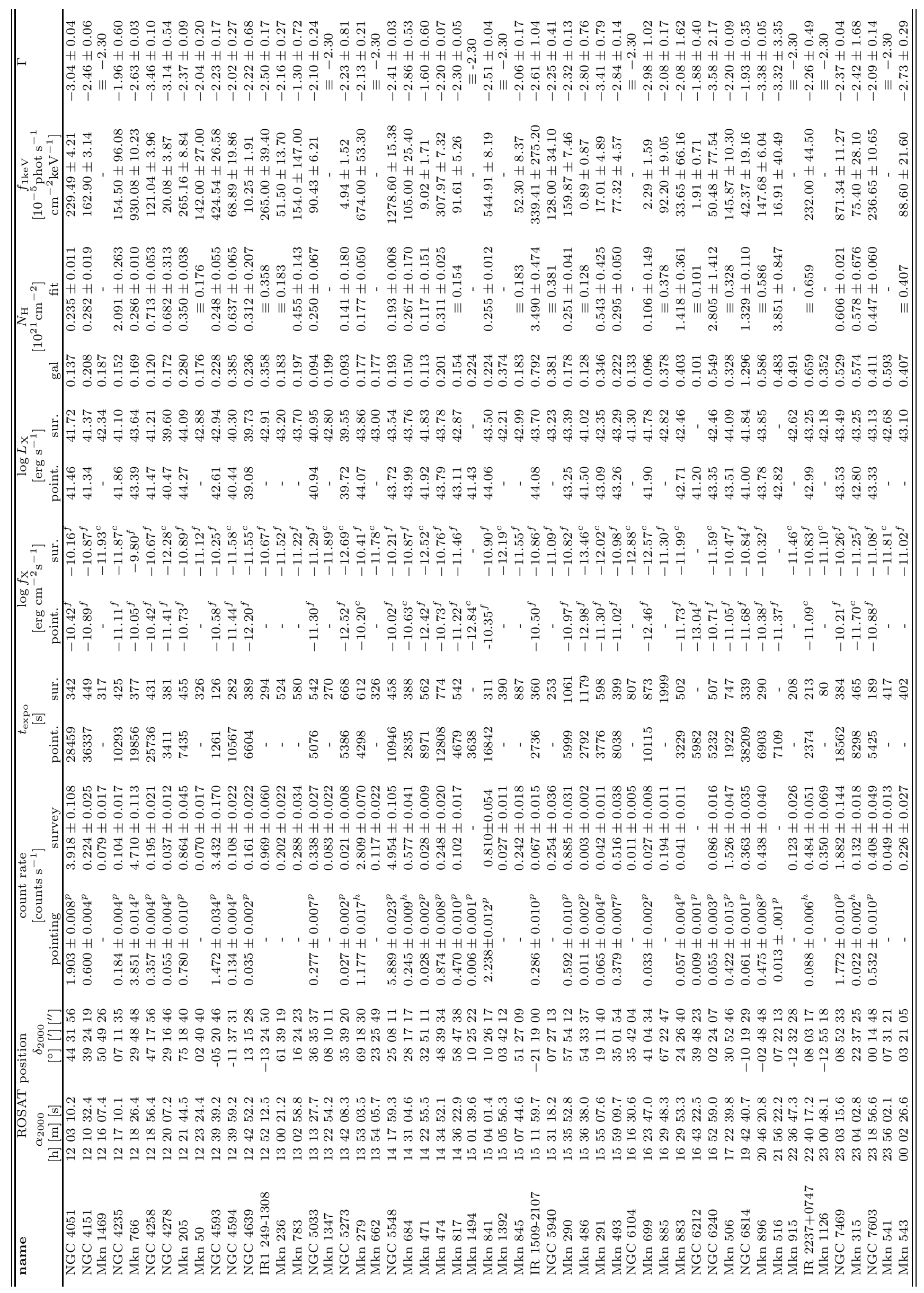


Table C.4. Seyfert2 galaxies: The table contains soft X-ray properties of the interacting or isolated Seyfert 2 galaxies. For the description of columns see Table C.3

\begin{tabular}{|c|c|c|c|c|c|c|c|c|c|c|c|c|c|c|}
\hline \multirow[t]{3}{*}{ name } & \multicolumn{2}{|c|}{ ROSAT position } & \multirow{2}{*}{\multicolumn{2}{|c|}{$\begin{array}{c}\text { count rate } \\
{\left[\text { counts s}^{-1}\right]}\end{array}$}} & \multirow{2}{*}{\multicolumn{2}{|c|}{$\begin{array}{c}t_{\text {expo }} \\
{[\mathrm{s}]}\end{array}$}} & \multirow{2}{*}{\multicolumn{2}{|c|}{$\begin{array}{c}\log f_{\mathrm{X}} \\
{\left[\mathrm{erg} \mathrm{cm}^{-2} \mathrm{~S}^{-1}\right]}\end{array}$}} & \multirow{2}{*}{\multicolumn{2}{|c|}{$\begin{array}{c}\log L_{\mathrm{X}} \\
{\left[\operatorname{erg~s}^{-1}\right]}\end{array}$}} & \multirow{2}{*}{\multicolumn{2}{|c|}{$\begin{array}{c}N_{\mathrm{H}} \\
{\left[10^{21} \mathrm{~cm}^{-2}\right]}\end{array}$}} & \multirow{3}{*}{$\begin{array}{c}f_{1 \mathrm{keV}} \\
{\left[10^{-5} \mathrm{phot} \mathrm{s}^{-1}\right.} \\
\left.\mathrm{cm}^{-2} \mathrm{keV}^{-1}\right]\end{array}$} & \multirow[t]{3}{*}{$\Gamma$} \\
\hline & $\alpha_{2000}$ & $\delta_{2000}$ & & & & & & & & & & & & \\
\hline & {$[\mathrm{h}][\mathrm{m}][\mathrm{s}]$} & {$\left[{ }^{\circ}\right]\left[\left[^{\prime}\right]\left[{ }^{\prime \prime}\right]\right.$} & pointing & survey & point. & sur. & point. & sur. & point. & sur. & gal & fit & & \\
\hline Mkn 348 & 004847.4 & 315716 & $0.010 \pm 0.001^{p}$ & - & 23437 & - & $-12.52^{f}$ & - & 41.06 & - & 0.591 & $0.644 \pm 0.334$ & $3.98 \pm 2.40$ & $-2.43 \pm 0.88$ \\
\hline IR 0135-1307 & 013805.4 & -125214 & $0.010 \pm 0.001^{p}$ & - & 6780 & - & $-11.41^{f}$ & - & 43.14 & - & 0.245 & $0.950 \pm 0.674$ & $2.41 \pm 0.99$ & $-4.36 \pm 1.23$ \\
\hline Mkn 573 & 014357.8 & 022051 & $0.076 \pm 0.002^{p}$ & $0.066 \pm 0.016$ & 13683 & 386 & $-11.40^{f}$ & $-11.88^{c}$ & 42.35 & 41.87 & 0.291 & $0.430 \pm 0.107$ & $15.85 \pm 1.62$ & $-3.32 \pm 0.24$ \\
\hline IR 0147-0740 & $\begin{array}{lll}01 & 50 & 02.7\end{array}$ & -072540 & $0.040 \pm 0.002^{p}$ & - & 6896 & - & $-11.71^{f}$ & - & 42.03 & - & 0.219 & $3.275 \pm 3.520$ & $43.43 \pm 42.20$ & $-1.77 \pm 1.69$ \\
\hline NGC 1068 & 024240.9 & -000043 & $1.957 \pm 0.040^{p}$ & $1.785 \pm 0.094$ & 1211 & 206 & $-9.83^{f}$ & $-10.26^{f}$ & 42.41 & 41.97 & 0.355 & $0.524 \pm 0.069$ & $478.49 \pm 28.30$ & $-3.44 \pm 0.14$ \\
\hline NGC 1144 & 025511.9 & -001043 & $0.007 \pm 0.001^{p}$ & & 11855 & 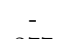 & $-12.07^{f}$ & & 42.16 & 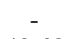 & 0.619 & $0.879 \pm 0.689$ & $2.69 \pm 0.97$ & $-3.46 \pm 1.14$ \\
\hline IR 0253-1641 & $02 \quad 56 \quad 01.7$ & -162919 & - & $0.093 \pm 0.021$ & - & 277 & - & $-11.70^{c}$ & - & 42.63 & 0.322 & - & - & $\equiv-2.30$ \\
\hline Mkn 1066 & 025958.7 & $3649 \quad 17$ & $0.005 \pm 0.001^{h}$ & & 15605 & & $-12.21^{c}$ & & 41.23 & & 1.185 & - & - & $\equiv-2.30$ \\
\hline Mkn 607 & 032449.0 & -030237 & - & $0.030 \pm 0.013$ & - & 341 & - & $-12.14^{c}$ & 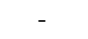 & 41.05 & 0.397 & - & - & $\equiv-2.30$ \\
\hline IR 0450-0317 & 045244.0 & -031256 & $0.007 \pm 0.001^{p}$ & . & 16025 & - & $-12.23^{f}$ & - & 41.47 & - & 0.437 & $0.794 \pm 0.729$ & $2.52 \pm 0.89$ & $-3.28 \pm 1.63$ \\
\hline NGC 2110 & 055211.6 & -072725 & $0.018 \pm 0.001^{h}$ & $0.037 \pm 0.010$ & 31439 & 510 & $-11.59^{c}$ & $-11.72^{c}$ & 41.39 & 41.25 & 1.731 & - & - & $\equiv-2.30$ \\
\hline Mkn 3 & 061535.6 & 710210 & $0.062 \pm 0.002^{p}$ & $0.056 \pm 0.014$ & 21098 & 393 & $-10.63^{f}$ & $-11.68^{c}$ & 42.95 & 41.89 & 0.846 & $2.350 \pm 1.190$ & $56.82 \pm 4.47$ & $-3.61 \pm 1.46$ \\
\hline Mkn 620 & $\begin{array}{lll}06 & 50 & 09.1\end{array}$ & 605048 & $0.001 \pm 0.000^{h}$ & - & 34465 & - & $-12.94^{c}$ & - & 39.90 & - & 0.731 & - & - & $\equiv-2.30$ \\
\hline Mkn 78 & 074241.2 & $65 \quad 1032$ & $0.009 \pm 0.001^{p}$ & 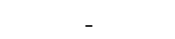 & 14697 & 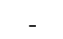 & $-11.22^{f}$ & - & 43.27 & - & 0.405 & $1.360 \pm 0.709$ & $4.08 \pm 1.11$ & $-4.32 \pm 1.32$ \\
\hline Mkn 1210 & $\begin{array}{llll}08 & 04 & 06.1\end{array}$ & $\begin{array}{llll}05 & 07 & 04\end{array}$ & - & \pm 0 & - & 272 & - & -12.2 & & 1.8 & & & & $\equiv-2.30$ \\
\hline NGC 3081 & 095930.3 & -224954 & - & $0.022 \pm 0.008$ & - & 511 & - & $-12.22^{c}$ & - & 40.75 & 0.454 & - & - & $\equiv-2.30$ \\
\hline Mkn 720 & 101737.6 & 065820 & - & $0.086 \pm 0.016$ & & 434 & & $-11.80^{c}$ & & 42.80 & 0.256 & - & - & $\equiv-2.30$ \\
\hline Mkn 34 & 103408.3 & 600155 & $0.006 \pm 0.002^{h}$ & - & 1427 & - & $-12.57^{c}$ & - & 42.15 & - & 0.070 & - & - & $\equiv-2.30$ \\
\hline NGC 3660 & 112332.4 & -083932 & & $0.061 \pm 0.016$ & & 355 & & $-11.84^{c}$ & $x$ & 41.53 & 0.383 & - & - & $\equiv-2.30$ \\
\hline NGC 3982 & 115628.0 & 550732 & $0.015 \pm 0.002^{p}$ & - & 3851 & - & $-12.29^{f}$ & - & 39.94 & - & 0.122 & $0.374 \pm 0.575$ & $2.24 \pm 1.30$ & $-3.26 \pm 1.38$ \\
\hline NGC 4388 & 122546.7 & 123946 & $0.049 \pm 0.002^{p}$ & - & 11639 & - & $-11.07^{f}$ & - & 42.02 & - & 0.263 & $1.018 \pm 0.376$ & $19.68 \pm 3.61$ & $-3.64 \pm 0.62$ \\
\hline NGC4922B & 130125.3 & 291843 & $0.003 \pm 0.001^{h}$ & - & 17170 & - & $-12.88^{c}$ & - & 41.17 & - & 0.098 & - & - & $\equiv-2.30$ \\
\hline NGC 4941 & 130413.2 & $\begin{array}{lll}-05 & 3304\end{array}$ & - & $0.035 \pm 0.015$ & - & 268 & - & $-12.20^{c}$ & - & 40.04 & 0.248 & - & - & $\equiv-2.30$ \\
\hline NGC 5005 & 131056.3 & 370323 & $0.089 \pm 0.003^{p}$ & $0.062 \pm 0.013$ & 9004 & 540 & $-11.30^{f}$ & $-12.17^{c}$ & 40.94 & 40.06 & 0.111 & $0.558 \pm 0.155$ & $27.44 \pm 2.97$ & $-3.12 \pm 0.31$ \\
\hline IR $1329+0216$ & 133152.2 & 020057 & $0.011 \pm 0.001^{p}$ & - & 9791 & - & $-12.09^{f}$ & - & 43.14 & - & 0.185 & $0.373 \pm 0.510$ & $1.12 \pm 0.90$ & $-3.93 \pm 1.44$ \\
\hline NGC 5252 & 133816.0 & 043231 & $0.003 \pm 0.001^{h}$ & 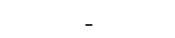 & 3745 & - & $-12.69^{c}$ & - & 41.28 & - & 0.197 & - & - & $\equiv-2.30$ \\
\hline Mkn 266SW & 133818.7 & $48 \quad 1642$ & $0.039 \pm 0.002^{p}$ & $0.043 \pm 0.011$ & 9807 & 562 & $-11.81^{f}$ & $-12.22^{c}$ & 42.38 & 41.96 & 0.174 & $0.410 \pm 0.177$ & $9.52 \pm 1.56$ & $-3.04 \pm 0.40$ \\
\hline NGC 5506 & 141315.0 & -031218 & $0.292 \pm 0.008^{p}$ & $0.106 \pm 0.019$ & 4380 & 366 & $-10.92^{c}$ & $-11.58^{c}$ & 42.05 & 41.40 & 0.405 & $>1.000$ & $4070 \pm 634$ & $\equiv-2.30$ \\
\hline Mkn 670 & $1414 \quad 17.5$ & 264441 & - & $0.061 \pm 0.013$ & - & 520 & - & $-12.11^{c}$ & - & 42.27 & 0.149 & - & - & $\equiv-2.30$ \\
\hline Mkn 673 & 141721.4 & 265141 & $0.008 \pm 0.001^{p}$ & - & 9205 & - & $-12.32^{f}$ & - & 42.10 & 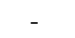 & 0.153 & $0.598 \pm 0.738$ & $2.33 \pm 1.05$ & $-3.18 \pm 1.27$ \\
\hline NGC 5929 & 152607.0 & 414016 & $0.013 \pm 0.001^{p}$ & - & 13356 & - & $-12.26^{f}$ & - & 40.83 & - & 0.208 & $0.716 \pm 0.498$ & $5.68 \pm 1.16$ & $-2.65 \pm 0.97$ \\
\hline NGC 5953 & 153432.5 & $15 \quad 1139$ & $0.008 \pm 0.001^{h}$ & $0.065 \pm 0.015$ & 10240 & 310 & $-12.25^{c}$ & $-11.84^{c}$ & 40.73 & 41.14 & 0.343 & - & - & $\equiv-2.30$ \\
\hline NGC 6211 & 164121.5 & 574624 & & $0.008 \pm 0.004$ & & 817 & the & $-12.90^{c}$ & & 40.99 & 0.192 & - & - & $\equiv-2.30$ \\
\hline NGC7319 & $22 \quad 36 \quad 03.2$ & 335836 & $0.003 \pm 0.001^{h}$ & - & 23326 & - & $-12.46^{c}$ & - & 41.51 & - & 0.773 & - & - & $\equiv-2.30$ \\
\hline NGC 7674 & 232757.0 & 084645 & $0.023 \pm 0.002^{p}$ & - & 3881 & - & $-10.91^{f}$ & - & 43.33 & - & 0.531 & $1.075 \pm 0.598$ & $6.94 \pm 2.19$ & $-4.41 \pm 1.01$ \\
\hline NGC 7743 & 234421.4 & 095557 & $0.004 \pm 0.001^{p}$ & - & 18904 & - & $-13.13^{f}$ & - & 39.84 & - & 0.525 & $\equiv 0.525$ & $1.59 \pm 0.74$ & $-1.83 \pm 1.48$ \\
\hline
\end{tabular}



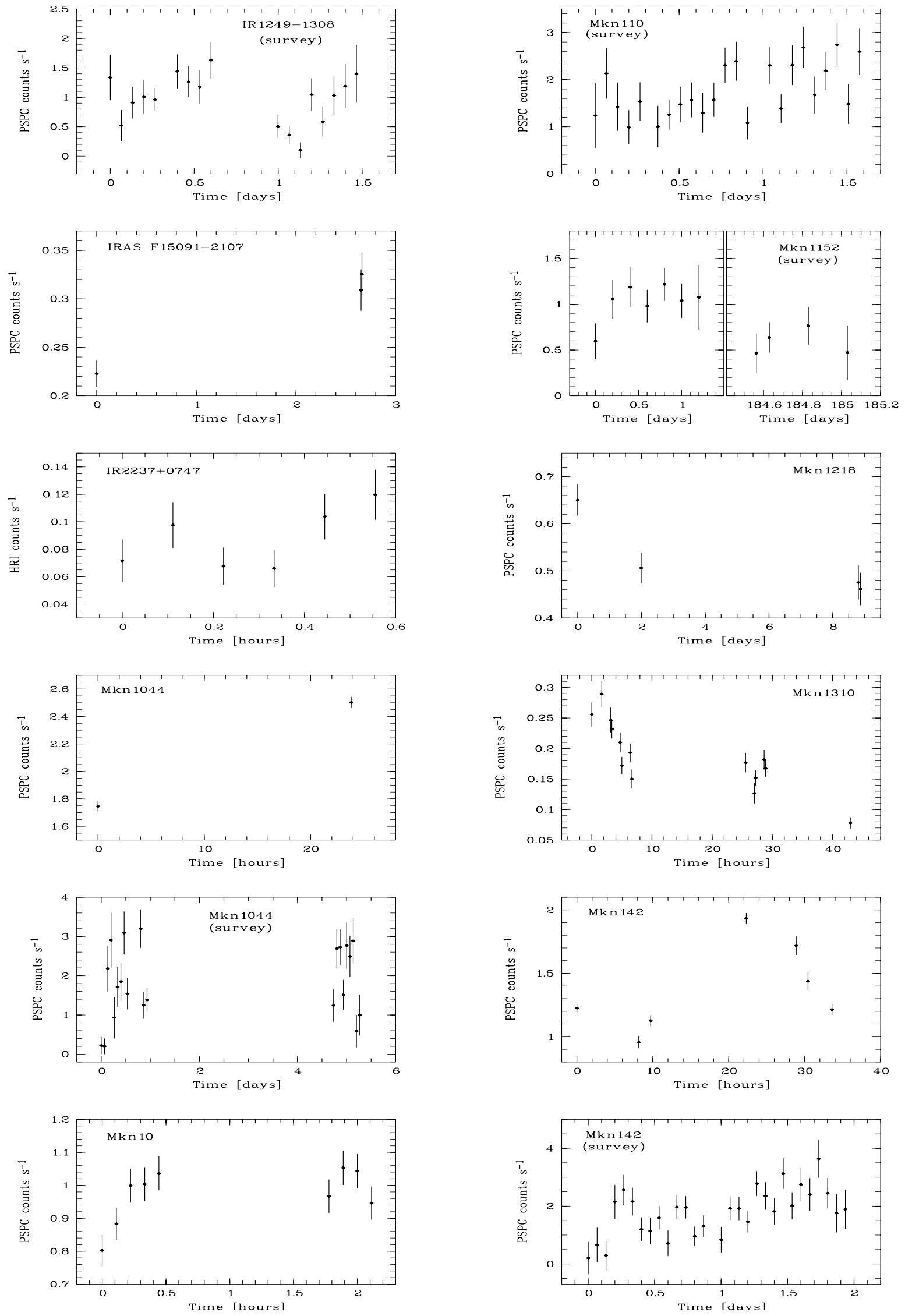

Fig. C.1. Light curves of variable Seyfert 1 galaxies 

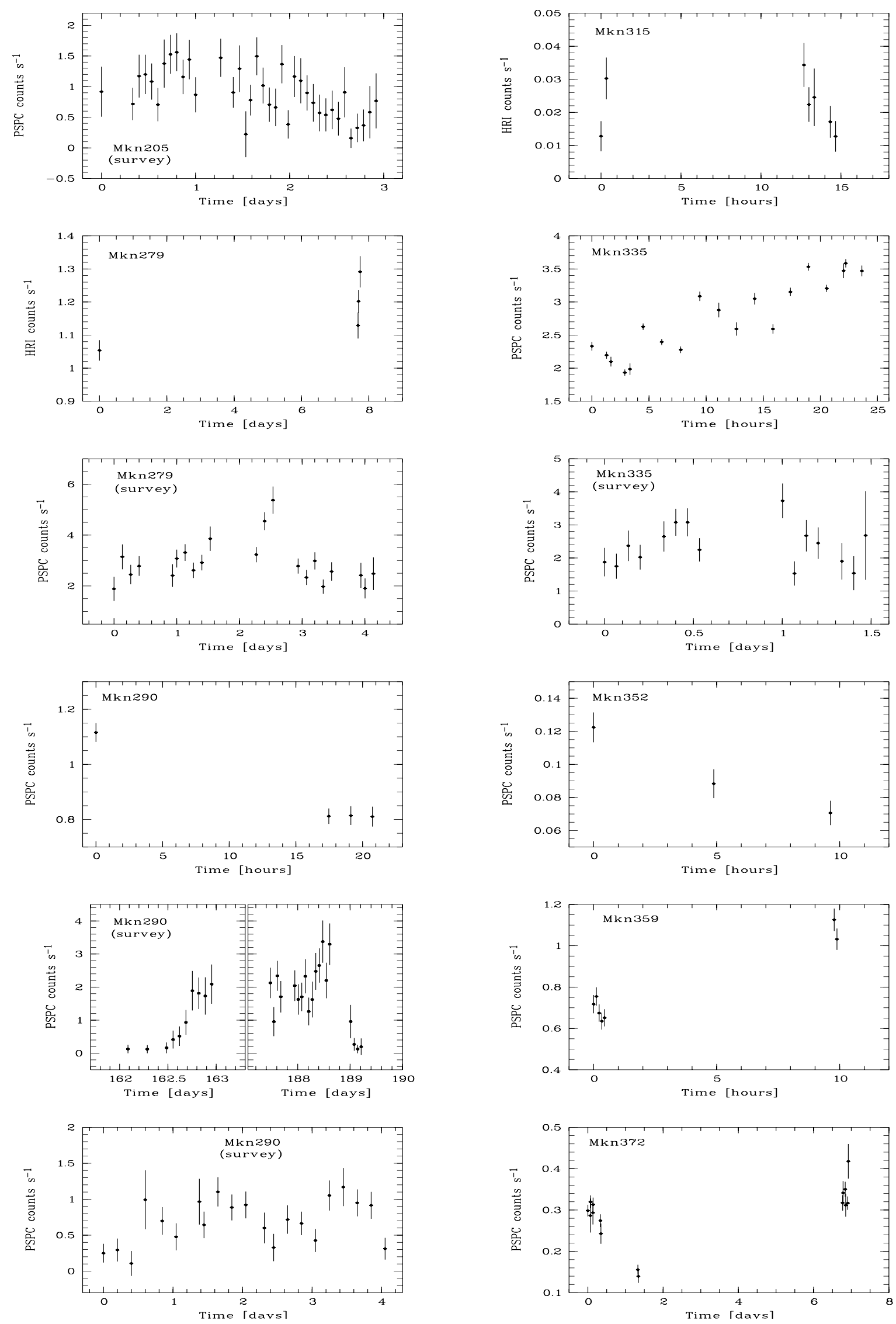

Fig. C.2. Light curves of variable Seyfert 1 galaxies 

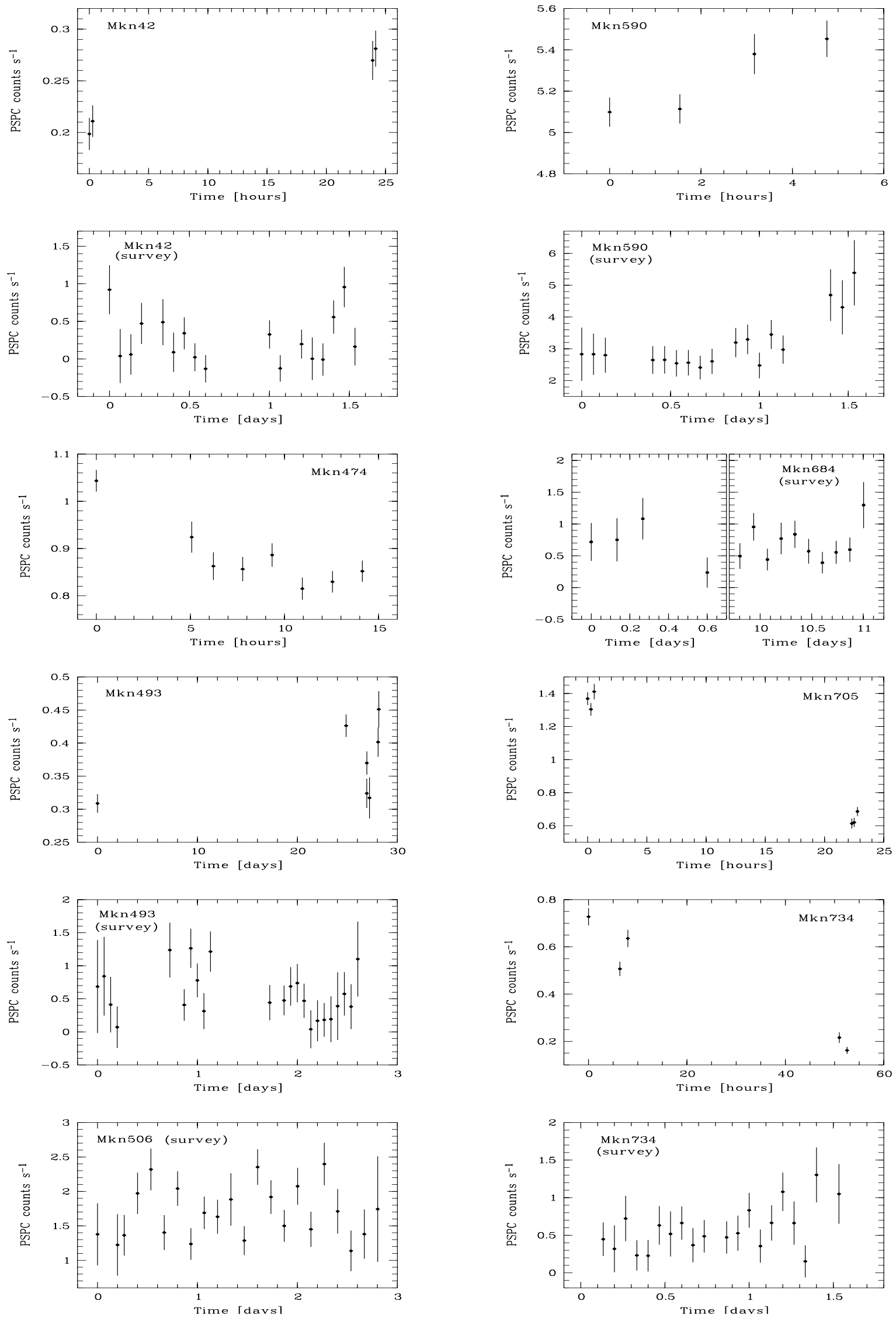

Fig. C.3. Light curves of variable Seyfert 1 galaxies 

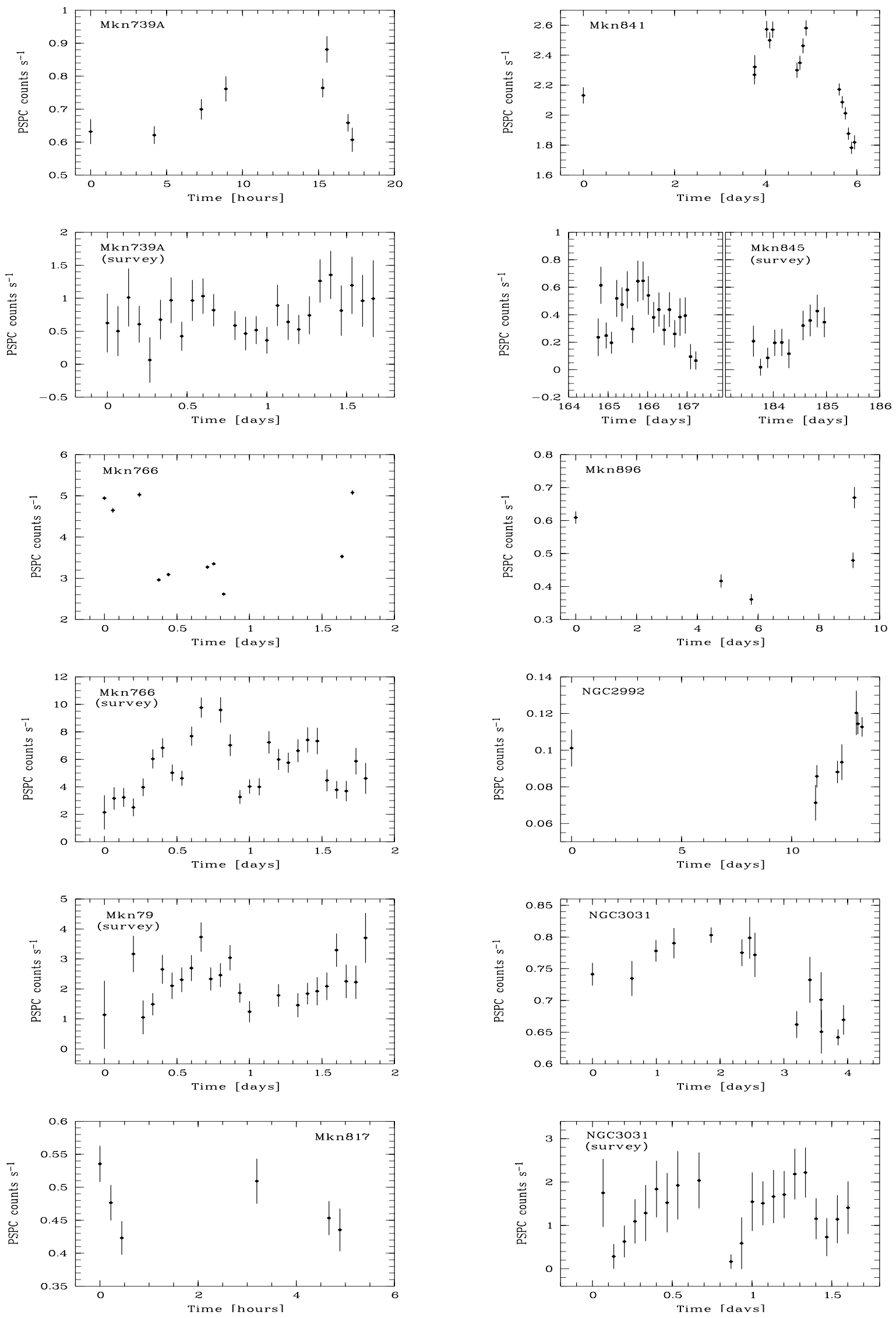

Fig. C.4. Light curves of variable Seyfert 1 galaxies 

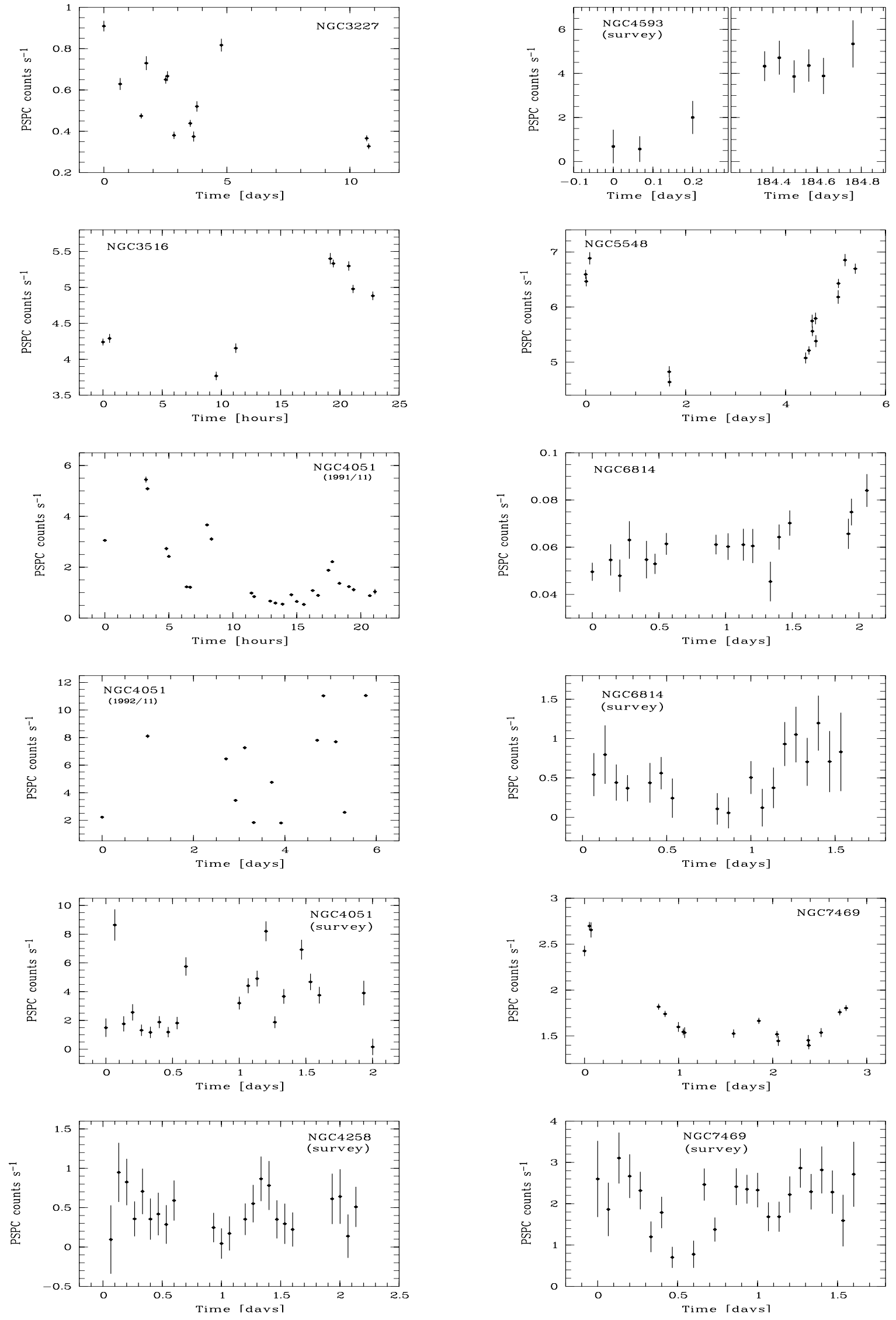

Fig. C.5. Light curves of variable Seyfert 1 galaxies 


\section{References}

Barnes, J. E., \& Hernquist, L. E. 1991, ApJ, 370, L65

Barnes, J. E., \& Hernquist, L. 1996, ApJ, 471, 115B

Bassani, L., Dadina, M., Maiolino, R., Salvati, M., et al. 1999, ApJS, 121, 473

Bernlöhr, K. 1993, A\&A, 268, 25

Boller, Th., Brandt, W. N., \& Fink, H. 1996, A\&A, 305, 53

Bushouse, H. A. 1987, ApJ, 320, 49

Dahari, O. 1984, AJ, 89, 966

Dickey, J. M., \& Lockman, F. J. 1990, ARA\&A, 28, 215

Frank, J., King, A. R., \& Raine, D. J. 1985, Accretion power in astrophysics (Cambridge University Press) 90

Freedman, W. L., et al. 1994, ApJ, 427, 628

Grupe, D. 1996, Properties of bright soft X-ray selected ROSAT AGN, Dissertation submitted Georg-August University, Göttingen, Germany

Georgantopoulos, I., \& Papadakis, I. E. 2000, MNRAS, submitted [astro-ph/0008295]

Hummel, E. 1981, A\&A, 96, 111

Helou, G., Soifer, B. T., \& Rowan-Robinson, M. 1985, ApJ, 298, L7

Jog, C. J., \& Das, M. 1992, ApJ, 400, 476

Jog, C. J., \& Solomon, P. M. 1992, ApJ, 387, 152

Kennicut Jr., R. C., Keel, W. C., van der Hulst, J. M., Hummel, E., \& Roettiger, K. A. 1987, AJ, 93, 1011
Lipovetski, V. A., Neizvestny, S. J., \& Neizvestnaya, O. M. 1987, A Catalogue of Seyfert Galaxies, Communications of the Special Astrophysical Observatory, 55

Mathur, S. 2000, MNRAS, 314, L17

Mihos, J. C., \& Hernquist, L. 1996, ApJ, 464, 641

Osterbrock, D. E., \& Pogge, R. W. 1985, ApJ, 297, 166

Pietsch, W., Trinchieri, G., Arp, H., \& Sulentic, J. W. 1997, A\&A, 322, 89P

Rafanelli, P., Violato, M., \& Baruffolo, A. 1995, AJ, 109, 1546

Rafanelli, P., Piro, L., Radovich, M., et al. 1997, Mem. della Soc. Astron. Ital., 68, 301

Rafanelli, P., Osterbrock, D. E., \& Pogge, R. W. 1990, AJ, 99, $53 \mathrm{R}$

Rubin, V. C., Ford, Jr. W. K., Thonnard, N., \& Burstein, D. 1982, ApJ, 261, 439

Schmidt, M., \& Green, R. F. 1986, ApJ, 305, 68

Smith, E. P., \& Kassim, N. E. 1993, AJ, 105, 46

Telesco, C. M., Dressel, L. L., \& Wolstencroft, R. D. 1993, ApJ, 414,120

Veron-Cetty, M. P., \& Veron, P. 1991, A Catalogue of Quasars and Active Nuclei, 5th ed. (ESO, Garching near Munich)

Voges, W., Aschenbach, B., Boller, Th., et al. 1999, A\&A, 349, 389

Walter, R., \& Fink, H. H. 1993, A\&A, 274, 105W

Zimmermann, H. U., Belloni, T., Izzo, C., Kahabka, P., \& Schwentker, O. 1994, MPE Report 257 